\title{
WALLABY Pre-Pilot Survey: H I Content of the Eridanus Supergroup
}

\author{
B.-Q. For, ${ }^{1,2 \star}$ J. Wang, ${ }^{3}$ T. Westmeier, ${ }^{1,2}$ O.I. Wong, ${ }^{4,1,2}$ C. Murugeshan, ${ }^{4,2}$
}

L. Staveley-Smith, ${ }^{1,2}$ H.M. Courtois,${ }^{5}$ D. Pomarède, ${ }^{6}$ K. Spekkens, ${ }^{7}$ B. Catinella,,${ }^{1,2}$

K.B.W. McQuinn, ${ }^{8}$ A. Elagali, ${ }^{9}$ B.S. Koribalski, ${ }^{10,11,2}$ K. Lee-Waddell, ${ }^{1,4}$ J.P. Madrid, ${ }^{12}$

A. Popping, ${ }^{1}$ T.N. Reynolds, ${ }^{1,2}$ J. Rhee, ${ }^{1,2}$ K. Bekki, ${ }^{1}$ H. Dènes, ${ }^{13}$ P. Kamphuis,${ }^{14}$ \&

L. Verdes-Montenegro ${ }^{15}$

${ }^{1}$ International Centre for Radio Astronomy Research, University of Western Australia, 35 Stirling Hwy, Crawley, WA 6009, Australia

${ }^{2}$ ARC Centre of Excellence for All Sky Astrophysics in 3 Dimensions (ASTRO 3D)

${ }^{3}$ Kavli Institute for Astronomy and Astrophysics, Peking University, Beijing 100871, China

${ }^{4}$ CSIRO Space \& Astronomy, PO Box 1130, Bentley, WA 6102, Australia

${ }^{5}$ Univ Claude Bernard Lyon 1, IP2I Lyon, IUF, F-69622, Villeurbanne, France

${ }^{6}$ Institut de Recherche sur les Lois Fondamentales de l'Univers, CEA Universit Paris-Saclay, France

${ }^{7}$ Royal Military College of Canada, PO Box 17000, Station Forces, Kingston, ON K7K7B4, Canada

${ }^{8}$ Rutgers University, Department of Physics and Astronomy, 136 Frelinghuysen Road, Piscataway, NJ 08854, USA

${ }^{9}$ Telethon Kids Institute, Perth Children's Hospital, Perth, Australia

${ }^{10}$ CSIRO Space \& Astronomy, PO Box 76, Epping, NSW 1710, Australia

${ }^{11}$ School of Science, Western Sydney University, Locked Bag 1797, Penrith, NSW 2751, Australia

${ }^{12}$ The University of Texas Rio Grande Valley, One West University Blvd, Brownsville, TX 78520, USA

${ }^{13}$ ASTRON, The Netherlands Institute for Radio Astronomy, Oude Hoogeveensedijk 4, 7991 PD, Dwingeloo, the Netherlands

${ }^{14}$ Ruhr University Bochum, Faculty of Physics and Astronomy, Astronomical Institute, 44780 Bochum, Germany

${ }^{15}$ Instituto de Astrofísica de Andalucía, CSIC, Glorieta de la Astronomía, E-18080, Granada, Spain

Accepted 2021 July 30. Received 2021 July 29; in original form 2021 July 5

\begin{abstract}
We present observations of the Eridanus supergroup obtained with the Australian Square Kilometre Array Pathfinder (ASKAP) as part of the pre-pilot survey for the Widefield ASKAP L-band Legacy All-sky Blind Survey (WALLABY). The total number of detected $\mathrm{H}$ I sources is 55, of which 12 are background galaxies not associated with the Eridanus supergroup. Two massive $\mathrm{H}_{\text {I }}$ clouds are identified and large $\mathrm{H}_{\mathrm{I}}$ debris fields are seen in the NGC 1359 interacting galaxy pair, and the face-on spiral galaxy NGC 1385. We describe the data products from the source finding algorithm and present the basic parameters. The presence of distorted $\mathrm{H}_{\mathrm{I}}$ morphology in all detected galaxies suggests ongoing tidal interactions within the subgroups. The Eridanus group has a large fraction of $\mathrm{H}$ I deficient galaxies as compared to previously studied galaxy groups. These $\mathrm{H}$ I deficient galaxies are not found at the centre of the group. We find that galaxies in the Eridanus supergroup do not follow the general trend of the atomic gas fraction versus stellar mass scaling relation, which indicates that the scaling relation changes with environmental density. In general, the majority of these galaxies are actively forming stars.
\end{abstract}

Key words: galaxies: star formation - galaxies: ISM - galaxies: groups: general

\section{INTRODUCTION}

The star formation-density and morphology-density relations highlight the dependence of galaxy evolution on environment. A galaxy cluster is the most striking place to look for environmental effects on galaxy evolution. Observations show that the star formation is suppressed in cluster environments (Gómez et al. 2003; Cortese et al. 2019) and the fraction of early-type (elliptical and lenticular) galaxies increases in denser cluster environments as compared to the fraction of late-type (spiral and irregular) galaxies (Dressler 1980; Postman et al. 2005). Recent studies of clusters in the local Universe have shown conflicting results. While a morphology-density relation re-

\footnotetext{
^ E-mail: biqing.for@uwa.edu.au
}

mains within each halo, the total fraction of early-type galaxies is nearly constant across three orders of magnitude in cluster halo mass $\left(13 \leq \log M_{\mathrm{h}} /\left(h^{-1} \mathrm{M}_{\odot}\right) \leq 15.8\right)$ (Hoyle et al. 2012). The study of Simard et al. (2009) also shows no clear trend of the early-type fractions in clusters as a function of cluster velocity dispersion. These results suggest that most morphological transformation is happening in groups prior to merging into massive clusters. The small velocity dispersion of galaxy groups also results in more galaxy mergers than in clusters (e.g., Hickson 1997). A combination of nature and nurture must be at play to drive such differences. The evolutionary effects imposed by different environments are important to understand.

In order to gain a full picture of galaxy evolution, it is crucial to investigate the gas and stellar content of galaxies. Hydrogen gas makes up the baryonic matter that is channeling through large-scale 
structure filaments to haloes, cooling to neutral form in galaxy disks and then to molecular gas to form stars. Observations of $\mathrm{H}_{\mathrm{I}}$ are a direct way to trace recent and ongoing interaction between galaxies and their environment due to its large extent beyond the stellar disk (Hibbard \& van Gorkom 1996). Interactions can occur via a number of physical processes, such as cold gas accretion with fresh gas channeling from the intergalactic medium (IGM) directly into the galaxies, hot mode gas accretion with gas being shock-heated to high halo virial temperatures and falling back to the galaxy centre as it cools (Katz et al. 2003; Kereš et al. 2005), tidal stripping due to gravitational interaction (Chung et al. 2009; For et al. 2014) and ram-pressure stripping when galaxies pass through a dense IGM (Kenney et al. 2004; Abramson et al. 2011). These processes largely contribute to gas removal and accretion in galaxies, which in turn, affect their star formation efficiency (Cortese et al. 2021).

There is evidence that galaxies have undergone "pre-processing" in group environments before falling into clusters (Zabludoff \& Mulchaey 1998; Mahajan 2013). Thus, galaxy groups are the ideal place for following the galaxy evolutionary path. In addition, galaxy groups are more common than clusters (Tully 2015). Examples include the studies of the NGC 3783 group (Kilborn et al. 2006), the Sculptor group (Westmeier et al. 2017), NGC 2997 and NGC 6946 (Pisano 2014) using single-dish telescopes as well as the Hickson compact groups using the interferometer (Verdes-Montenegro et al. 2001). Systematic surveys of a large sample of galaxy groups are not an easy task in part due to the trade-off between angular resolution and sensitivity. Single-dish telescopes provide excellent sensitivity but at arcminutes angular resolution. In order to resolve gas disks and $\mathrm{H}_{\mathrm{I}}$ debris, high angular resolution imaging with the use of interferometers is required. However, the required observing time is significantly more for an interferometer to reach the equivalent low $\mathrm{H}$ I column density sensitivity of a single-dish telescope. In addition, a traditional interferometer has a small field of view (FOV), which makes targeting clusters a better option for providing large samples than galaxy groups for the equivalent amount of observing time.

The Widefield ASKAP L-Band Legacy All-sky Blind SurveY (WALLABY, Koribalski et al. 2020) makes use of the large FOV capability of the Australian Square Kilometre Array Pathfinder (ASKAP; Johnston et al. 2007) to image $\mathrm{H}_{\mathrm{r}}$ galaxies out to a redshift $z \sim 0.26$ and across $3 \pi$ steradian of the sky $\left(-90^{\circ}<\delta<+30^{\circ}\right)$. The estimated root-mean-square (RMS) noise level is anticipated to be $1.6 \mathrm{mJy}$ per beam per $18.5 \mathrm{kHz}$ channel. The survey is expected to detect 500,000 galaxies (Koribalski et al. 2020). The main advantage of ASKAP over traditional radio interferometers is the use of a state-of-the-art phased array feed (PAF), which allows the formation of multiple beams on the sky simutaneously to achieve a fast survey speed. For WALLABY, the targeted angular and spectral resolution of $30^{\prime \prime}$ and $4 \mathrm{~km} \mathrm{~s}^{-1}$ at $z=0$ are two to thirty times better than some of the single-dish surveys, most notably the H I Parkes All-Sky Survey (HIPASS; Barnes et al. 2001) and the Arecibo Legacy Fast ALFA survey (ALFALFA; Giovanelli et al. 2005). WALLABY will revolutionize the $\mathrm{H}_{\mathrm{I}}$ extragalactic field by providing a large statistical sample of galaxies at high resolution across a wide range of environments from voids to groups and clusters.

To verify the observational feasibility of ASKAP and to improve the efficiency of the ASKAP data reduction pipeline, a WALLABY early science program was carried out in 2018 using 12 to 16 ASKAP antennas. Studies of the NGC 7162 group (Reynolds et al. 2019) and the LGG 351 group of Galaxies 351 (For et al. 2019) show that the majority of galaxies in these groups are actively forming stars indicating inefficiency of gas removal processes in the loose group environment. $\mathrm{H}_{\mathrm{I}}$ debris due to tidal interaction has also been identified

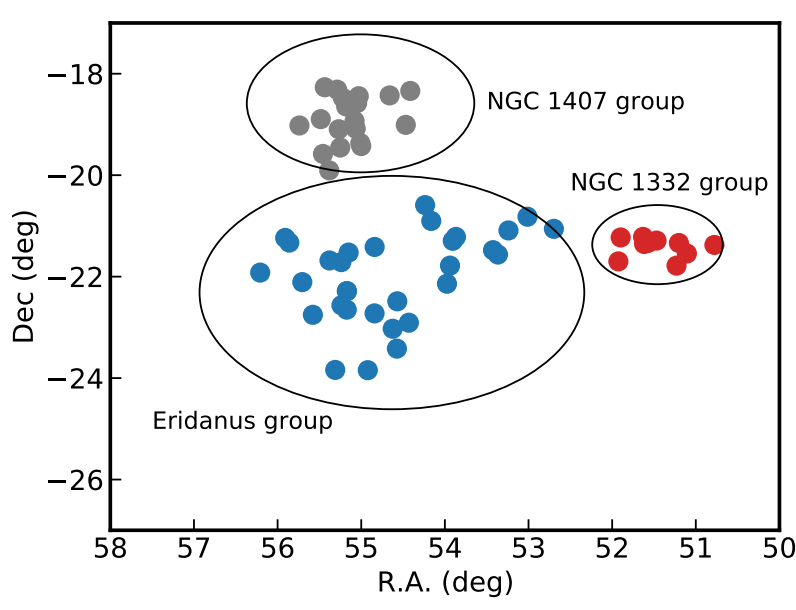

Figure 1. Distribution of galaxies in the Eridanus supergroup. Group members of NGC 1407, NGC 1332 and Eridanus groups as identified in Brough et al. (2006) are represented by grey, red and blue dots, respectively. The ellipses indicate that maximum radial extent of the groups.

in the NGC 7232 galaxy group, with one gas cloud postulated to be the progenitor of a long-lived tidal dwarf galaxy (Lee-Waddell et al. 2019). Detailed studies of individual galaxies, NGC 1566 (Elagali et al. 2019) and IC 5201 (Kleiner et al. 2019), have also highlighted the importance of high resolution imaging. The resolved $\mathrm{H}$ I kinematics allowed them to recover the dark matter fractions by modelling the observed rotational curves and to investigate the asymmetries of H I morphology of these galaxies.

\subsection{Eridanus Supergroup}

Studies of the Eridanus concentration/region can be traced back to 1930s (see Baker 1933). A later in-depth study by de Vaucouleurs (1975) found that Group 31 along with galaxies associated with NGC 1332 and NGC 1209 formed the "Eridanus Cloud". This cloud lies on the Eridanus-Fornax-Dorado filamentary structure and is connected to the "Great Wall" feature at $\sim 4000 \mathrm{~km} \mathrm{~s}^{-1}$ in the background (da Costa et al. 1988; Willmer et al. 1989). Several studies have shown the complex nature of the region. Willmer et al. (1989) showed that the region is made up of three or four subclumps, which are dynamically bound to each other and condensing to form a cluster. On the other hand, Omar \& Dwarakanath (2005a) considered that the galaxies in the region as loose groups and in an intermediate evolutionary stage between the Ursa-Major group and the Fornax cluster. A re-analysis of this region using the $6 \mathrm{dF}$ Galaxy Survey (6dFGS; Jones et al. 2004) suggests that there are 3 distinct groups, namely the NGC 1407, NGC 1332 and Eridanus groups (Brough et al. 2006, hereafter B06). These groups also form part of the supergroup, which is defined as a group of groups that may eventually merge to form a cluster. Figure 1 shows the group members as identified in Brough et al. (2006).

The Eridanus supergroup is chosen as a pre-pilot field because it presents a different environment than the early science fields, which allows us to probe the subcluster merging/pre-processing scenario. It is thought to be an intermediate step of evolutionary path for clusters assembly, similar to the Ursa Major Supergroup (Wolfinger et al. 2016). Our goals of this study are to investigate (1) the $\mathrm{H}$ i content of galaxies in the Eridanus field; (2) if the galaxies follow the typical $\mathrm{H}_{\mathrm{I}}$ scaling relation; (3) if their star formation rates reveal any difference 
in the merging/pre-processing environment, (4) if they are deficient in $\mathrm{HI}_{\mathrm{I}}$ and (5) if the $\mathrm{HI}_{\mathrm{I}}$ deficiency parameter correlates with the projected distance from the group centre.

This paper is structured as follows. In Section 2, we describe the previous $\mathrm{H}_{\mathrm{I}}$ observations, ASKAP observations, its data reduction and quality assessment. In Section 3, we describe the source finding methodology, present the H i spectra, moment maps, mask and S/N maps, as well as compare the WALLABY integrated fluxes with pre-

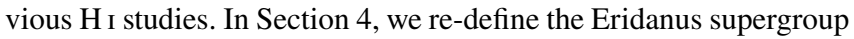
membership. In Section 5, we derive physical parameters: recession velocity, distance, $\mathrm{H}_{\mathrm{I}}$ linewidth, stellar mass, $\mathrm{H}_{\mathrm{I}}$ mass and $\mathrm{H}_{\mathrm{I}}$ deficiency parameter. In Sections 6 and 7, we compare the derived quantities with the $\mathrm{H}$ I scaling relation and star forming main-sequence. In Sections 8 and 9, we show a 3-dimensional model of the environment the Eridanus supergroup resides in and a summary and conclusions. We also describe the $\mathrm{H}_{\mathrm{I}}$ and optical morphologies in Appendix A.

Throughout the paper, we adopt a $\Lambda$ cold dark matter cosmology model $(\Lambda \mathrm{CDM})$ with $\Omega_{\mathrm{M}}=0.27, \Omega_{\mathrm{K}}=0, \Omega_{\Lambda}=0.73$ and $H_{0}=73$ $\mathrm{km} \mathrm{s}^{-1} \mathrm{Mpc}^{-1}$. These are the default parameters for distances and cosmological corrected quantities in the NASA/IPAC Extragalactic (NED) interface (Spergel et al. 2007).

\section{DATA}

\subsection{Previous H I Observations}

The Eridanus field has previously been observed as part of the singledish Parkes $\mathrm{H}_{\mathrm{I}}$ blind basketweave (BW) survey, with a follow-up study using the Australia Telescope Compact Array (ATCA; Waugh 2005) and one using the Giant Metrewave Radio Telescope (GMRT; Omar \& Dwarakanath 2005a,b). The BW survey of the Eridanus field covered $\sim 100 \mathrm{deg}^{2}$ and is centred on $\alpha=3^{\mathrm{h}} 22^{\mathrm{m}} 00^{\mathrm{s}}$ (J2000), $\delta=$ $-22^{\circ} 00^{\prime} 00^{\prime \prime}$. The BW scanning technique was aimed at achieving higher sensitivity than HIPASS, with RMS noise level a factor of 2 better (i.e., $\sim 7 \mathrm{mJy}$ ). The Hanning-smoothed velocity resolution is $26.4 \mathrm{~km} \mathrm{~s}^{-1}$. The follow-up ATCA observations targeted $24 \mathrm{H} \mathrm{I}$ sources with a median RMS of $6.5 \mathrm{mJy}$ and a sythesized beam of $\sim 113^{\prime \prime} \times 296^{\prime \prime}$. Some of the H I sources remained unresolved. The GMRT also targeted the Eridanus supergroup with 46 scientifically usable observations out of the 57 observed targets. The primary beam of the GMRT observation is $\sim 24^{\prime}$. The GMRT final image cubes are either low $\left(\sim 50^{\prime \prime}\right)$ or high resolution $\left(25^{\prime \prime}\right.$ or $\left.30^{\prime \prime}\right)$ with a velocity resolution of $\sim 13.4 \mathrm{~km} \mathrm{~s}^{-1}$. The $3 \sigma \mathrm{H}$ I column density detection limit is $\sim 1 \times 10^{20} \mathrm{~cm}^{-2}$ for the GMRT high resolution images.

\subsection{ASKAP Observations}

ASKAP is a radio interferometer located in the remote outback of Western Australia and is part of the Murchison Radio-astronomy Observatory (MRO). It consists of $36 \times 12$-m antennas, with each antenna equipped with a second-generation MK II PAF. ASKAP is designed to provide an instananeous large field of view $\left(5.5^{\circ} \times 5.5^{\circ}\right)$ with 36 dual-polarisation beams, a bandwidth of $288 \mathrm{MHz}$ as well as high angular and spectral resolutions (McConnell et al. 2016; Hotan et al. 2021). During the interim period between the ASKAP early science program and the pilot survey, WALLABY carried out a pre-pilot survey targeting the Eridanus field in March 2019. The survey attempted to utilise the full 36 antennas for the two interleaving footprints (footprints A and B) in contrast to the 12 to 16 antennas and the multiple observations per footprint for the early science program.

Each footprint has a $6 \times 6$ beam pattern and was rotated by $45^{\circ}$

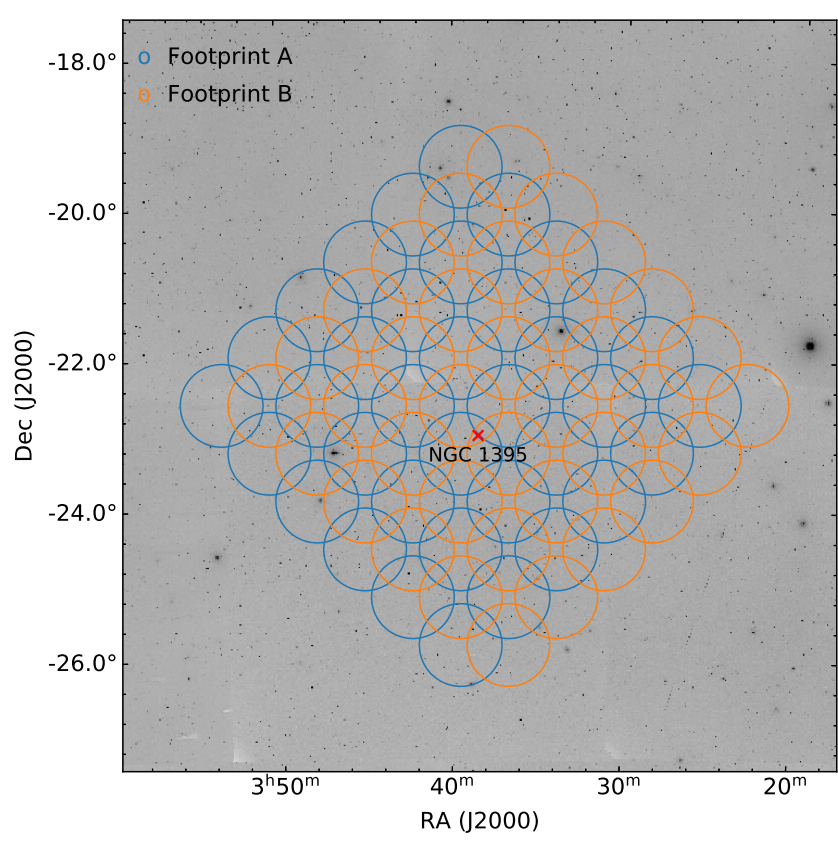

Figure 2. ASKAP footprints for the observations as listed in Table 1 overlaid on to the optical DSS-2 red image. The observed footprints have been rotated by position angle of $45^{\circ}$ relative to a standard ASKAP footprint. Each beam has an FWHM of $1^{\circ}$. The blue and orange circles represent footprint A and B, respectively. The brightest elliptical galaxy, NGC 1395, in the Eridanus supergroup is marked with cross.

on the sky. These observations were carried out during the day for footprint A and mostly at night time for footprint $\mathrm{B}$. The primary calibrator, PKS 1934-638, was observed for about $2 \mathrm{~h}$ at the beginning of each observation. Each observation is given a scheduling block identification number $\left(\mathrm{SBID}^{1}\right)$ and the total integration time for both footprints is $10.8 \mathrm{~h}$. We present the observing log in Table 1 and the on-sky positions of interleaved footprints in Figure 2.

\subsection{Data Reduction and Quality Assessment}

The data are processed automatically using ASKAPSOFT version 0.24.7 (Whiting 2020; Wieringa et al. 2020) installed on the "Galaxy" supercomputer at the Pawsey Supercomputing Centre. Only baselines shorter than $2 \mathrm{~km}$ and half of the full $288 \mathrm{MHz}$ bandwidth (i.e., 1295.5-1439.5 MHz) are processed. This is a huge improvement in data processing capability as compared to the early science program, in which only a small frequency range and few beams were processed at a given time (see e.g., For et al. 2019).

All 36 beams are used for bandpass calibration, and the autocorrelation of each beam measurement set is flagged out completely. Radio frequency interference (RFI) and antenna flagging are performed on a beam-by-beam basis. The overall flagged visibility fraction ranges from 10 to 30 percent across all beams and the mininum number of utilised antennas per beam is 31 . Subsequently, the derived bandpass solution is applied to each beam. Flux density calibration in each frequency channel is performed using PKS B1934-638 and gain calibration is performed via self-calibration. Continuum images of each beam are used as models to subtract continuum sources in the

1 The SBID can be used to search for the corresponding data set in CSIRO ASKAP Science Data Archive (CASDA). 
Table 1. ASKAP Observations log of the Eridanus Field.

\begin{tabular}{cccccccc}
\hline $\begin{array}{c}\text { UT date } \\
\text { (yyyy-mm-dd) }\end{array}$ & Footprint & $\begin{array}{c}\text { R.A. } \\
(\mathrm{h}: \mathrm{m}: \mathrm{s})\end{array}$ & $\begin{array}{c}\text { Decl. } \\
\left({ }^{\circ}{ }^{\prime \prime}\right)\end{array}$ & Calibrator SBID & $\begin{array}{c}\text { Science SBID } \\
\text { (Integration time } \\
(\mathrm{h})\end{array}$ & $\begin{array}{c}\text { Bandwidth } \\
(\mathrm{MHz})\end{array}$ & $\begin{array}{c}\text { Central frequency } \\
(\mathrm{MHz})\end{array}$ \\
\hline $2019-03-13$ & A & $3: 39: 30$ & $-22: 30: 00$ & 8169 & 8168 & 5.8 & 288 \\
$2019-03-13$ & B & $3: 36: 44.52$ & $-22: 37: 54.69$ & 8169 & 8170 & 5.0 & 288 \\
\hline
\end{tabular}

$u v$-domain. For the final spectral line imaging, a pixel size of $6^{\prime \prime}$ is adopted. We use Wiener filtering with a robustness of 0.5 in addition to Gaussian tapering to a resolution of $\sim 30^{\prime \prime}$. Multi-scale CLEAN is used to deconvolve the image (Cornwell 2008). Once the spectral line image cube per beam is created, image based continuum subtraction of the residual continuum emission is performed by fitting a low-order polynomial to each spectrum. Finally, we create a primary beam corrected beam-by-beam mosaic image cube. Channels in beams that are severely affected by artefacts have been masked out in the image cube. A particular type of artefact in the form of large-scale stripes across the beam is found to have been caused by deconvolution failing during the imaging step. The origin of the divergence comes from over-flagging of channels that contain Galactic emission.

We evaluate the data quality of each footprint image cube based on a set of metrics. These metrics are established based on the data in the WALLABY early science field of M 83 (For et al. 2019), and include RMS, minimum and maximum flux densities, 1 percentile noise level and median absolute deviation of median flux (MADMF). Two sets of these values are calculated and recorded during the pipeline processing. Each set consists of values for three types of image cubes, i.e., before and after continuum subtraction image cubes as well as a residual image cube. The first and second sets give values for each channel of the mosaicked image cubes and for each channel of each beam, respectively. The RMS values of the footprint A and B mosaics increase as a function of frequency $(5-6 \mathrm{mJy})$, which is consistent with the theoretical RMS trend. The RMS values of combined mosaic across all beams vary from 2.4 to $4.4 \mathrm{mJy}$ with central beams having the lowest RMS noise level.

To evaluate the effect of broadband RFI/artefacts, we examine the median absolute deviation of maximum flux density (MADMFD) of each beam. This metric is sensitive to strong artefacts. In Figure 3, we show the variation of MADMFD, and the largest values correspond to the beams with artefacts. We also examine the distribution of flux density values for all voxels in each beam at the 1 percentile level. Excessive negative flux density is shown to be related to bandpass calibration and/or sidelobe issues. We fit each distribution with a Gaussian and use the variance as a metric to determine if beams have been affected by those issues. The metric shows a similar result to that of MADMFD for this data set.

A stand-alone script ${ }^{2}$ is used to generate an HTML style summary report for each SBID. This report provides basic observation information, statistical plots generated from the pipeline, plots for visibilities and antenna flagging statistics etc. The report of each footprint is included along with the data release on CASDA.

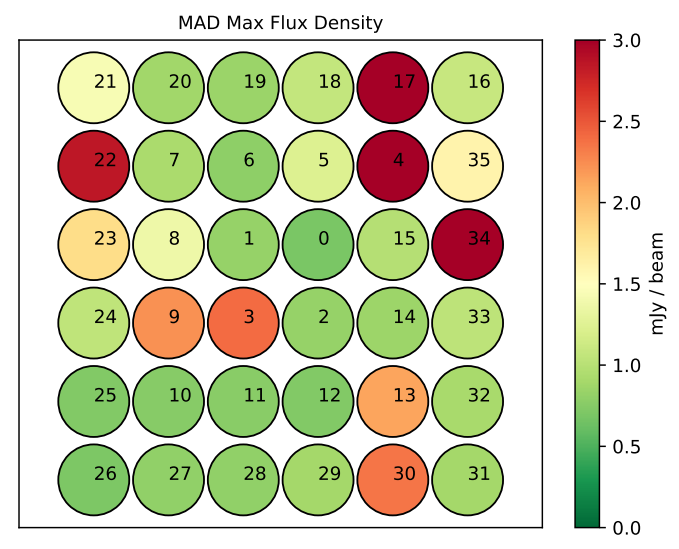

Figure 3. Median absolute deviation of maximum flux density (MADMFD) for each beam over frequency range $1295.5-1439.48 \mathrm{MHz}$ for footprint A. Beam numbers for the ASKAP $6^{\circ} \times 6^{\circ}$ footprint are labeled.

\section{SOURCE FINDING AND CATALOGING}

We use the Source Finding Application (sofiA ${ }^{3}$; Serra et al. 2015) version 2.0 (Westmeier et al. 2021) to search for $\mathrm{H}_{\mathrm{I}}$ sources in the mosaicked cube from $c z=500$ to $8500 \mathrm{~km} \mathrm{~s}^{-1}$. Channels that contain the Milky Way emission were excluded. Sofia was set to automatically flag some known artefacts, such as continuum residuals or RFI, prior to the search. We scale the data by the local RMS noise level and apply a threshold of $3.5 \sigma$ for the smooth + clip source detection algorithm. A radius of 2 pixels and 2 spectral channels are used to merge the detected voxels into objects. SOFIA generates a cubelet, an associated mask cube, a signal map with the total number of $\mathrm{H}_{\mathrm{I}}$ detected channels for each pixel, a spectrum, integrated $\mathrm{H}_{\mathrm{I}}$ intensity (0th moment), velocity field (1st moment) and velocity dispersion (2nd moment) maps for each detected source. To filter out false positive sources, sofia has implemented a reliability filter. The filter works by comparing the density of detections with positive and negative flux in parameter space to estimate the reliability of all positive detections under the principal assumption that astronomical sources must have positive flux, while the stochastic noise and any artefacts present in the data are symmetric about zero. A user-defined reliability threshold can then be applied to discard unreliable detections. We refer the reader to Serra et al. (2012) for a detailed description of the reliability filter.

The final catalogue consists of $55 \mathrm{H}$ i sources including two new H I sources (WALLABY J033911-222322 and WALLABY J033723235753; see Wong et al. 2021) that do not have optical counterparts in DR8 DESI Legacy Imaging Survey. The membership of each source is discussed in Section 4. In Figure 4, we present the integrated $\mathrm{H}_{\mathrm{I}}$

\footnotetext{
3 Available at https://github.com/SoFiA-Admin/SoFiA
}

2 Available at https://github.com/askap-qc/validation/blob/ master/wallaby_hi_val.py 
intensity map of individual detected sources enlarged by a factor of 4.5 in the Eridanus supergroup and the full mosaic with all sources labelled by their designated catalogue identification number (ID) (refer to Table 3). Within the WALLABY Eridanus FOV, 38 galaxies were also observed by the GMRT. Among those, 22 are detected by both WALLABY and GMRT. The remaining 16 non-detections are detected by GMRT but not by ASKAP. As a blind survey, WALLABY recovers an additional $19 \mathrm{H}$ I sources that were not targeted in the GMRT study. The properties and parameters of each source are given in Tables 3 and 4.

\section{1 $\mathrm{H}_{\mathrm{I}}$ intensity, velocity field, $\mathrm{S} / \mathrm{N}$ maps and $\mathrm{H}_{\mathrm{I}}$ spectra}

We convert the $\mathrm{H}$ I intensity maps ( $\mathrm{Jy} \mathrm{Hz}$ ) from sofia to integrated H I column density $\left(N_{\mathrm{HI}}\right)$ maps by using Eq. 76 in Meyer et al. (2017). We also create $1 \sigma$ flux density sensitivity maps by using the channel cubes from sofia. Subsequently, we divide each integrated $\mathrm{H}_{\mathrm{I}}$ column density map by its corresponding $1 \sigma N_{\mathrm{HI}}$ sensitivity map to obtain a signal-to-noise ratio $(\mathrm{S} / \mathrm{N})$ map. The $\mathrm{S} / \mathrm{N}$ ratio maps are used to clip the velocity field maps, in which pixels below a certain $\sigma$ are masked out. The spectrum of each source is created by integrating the flux densities of all spatial pixels in each spectral channel (provided by sofIA). Figure 5 shows the $N_{\mathrm{HI}}$ contours overlaid onto the DR8 DESI Legacy Imaging Survey $g$-band co-added image ${ }^{4}$ (refer to section 5.3 for detail), clipped velocity field maps and spectra in $V=\mathrm{c} z$, where $\mathrm{c}$ is the speed of light and $z$ is the $\mathrm{H}$ I derived redshift. Most of the detected galaxies are marginally resolved. Using these maps and images, we describe the $\mathrm{H}_{\mathrm{I}}$ and optical morphologies of individual galaxies in Appendix A.

\section{2 $\mathrm{H}_{\mathrm{I}}$ integrated flux}

To obtain the integrated flux $\left(S_{\text {int }}\right)$ in Jy Hz, we sum the flux densities across all channels within the mask. To verify the measured $S_{\text {int }}$ from WALLABY and for direct comparison with previous studies, we convert the values from Jy $\mathrm{Hz}$ to $\mathrm{Jy}_{\mathrm{km} \mathrm{s}}{ }^{-1}$. In Figure 6, we show the $S_{\text {int }}$ comparison between BW and HIPASS (Meyer et al. 2004) catalogues (top panel), the $S_{\text {int }}$ comparison between WALLABY and ATCA (middle panel), and the $S_{\text {int }}$ comparison between WALLABY and the single-dish studies (bottom panel). The solid line represents the unity line. Overall, the $S_{\text {int }}$ from two Parkes surveys are consistent with each other. For the interferometers comparison, there are outliers at the fainter end ( $<10 \mathrm{Jy} \mathrm{km} \mathrm{s}{ }^{-1}$ ). The $S_{\text {int }}$ values of fainter sources from ATCA are about a factor of two smaller than WALLABY's. We find that the $S_{\text {int }}$ values of these outliers are also a factor of two lower than BW, which suggests that the issue is not related to WALLABY. However, there does appear to be some missing flux in the ASKAP/ATCA interferometer results relative to both Parkes studies.

For the outlier, NGC 1415, a clear systematic offset is seen between the WALLABY and single-dish data. For NGC 1415, we verify that HIPASS has only detected half of the galaxy. The missing fluxes for some of the bright WALLABY sources can be explained. For example, NGC 1359 is an interaction galaxy pair, which WALLABY has resolved into tidal debris (see Figure 5). The diffuse emission is likely resolved out in this case. Both NGC 1398 and NGC 1359 are located in edge beams, where the noise level is significantly higher than in central beams. NGC $1367 / 1371$ is a large galaxy with an $\mathrm{H}_{\mathrm{I}}$

\footnotetext{
4 Co-added cutout images are available at https://datalab.noao.edu/ ls/dataAccess.php
}

diameter $\left(d_{\mathrm{HI}}\right)$ as observed by the Green Bank Telescope (GBT) of 22.3' (Sorgho et al. 2019). Our 0th-moment map shows a $d_{\mathrm{HI}}$ of $9.9^{\prime}$, which indicates that our observation does not recover the extended $\mathrm{H}$ I disc of this galaxy. We cannot make a comparison with the GMRT study because they also lost flux for large galaxies due to inadequate sampling of short spacing data.

We further investigate the missing flux issue by comparing the non-confused continuum sources with the NRAO VLA Sky Survey (NVSS; Condon et al. 1998). We find that our sources are systematically $6 \%$ lower in flux density than NVSS. However, the above issues (flux density calibration, sensitivity and zero spacing) cannot fully account for the offset between WALLABY and single-dish data. Results from the Rapid ASKAP Continuum Survey (RACS; McConnell et al. 2020) have shown position-dependent variations of flux density due to inappropriate primary beam corrections. To circumvent this issue, holography beam measurements will be used as part of the instrumental flux calibration for future observations.

To estimate the systematic offset in $\mathrm{H}_{\text {I }}$ fluxes, we compare the WALLABY data with all the single-dish data and fit an orthogonal distance regression (ODR) model. Both WALLABY and single-dish data errors are weighted for the ODR model (dashed line). We find a $\sim 20 \%$ mean difference. For statistical purposes, we use this mean correction factor for the derived parameters for all sources for the rest of the paper. We also list the uncorrected $S_{\text {int }}$.

\section{GROUP MEMBERSHIP}

By examining the older (Garcia et al. 1993; Garcia 1993; hereafter G93 for both) and newer (Tully 2015; Tully et al. 2016; hereafter T15 and TCS16, respectively) galaxy group catalogues, we find that several galaxies are not included as part of the groups in B06. This is in part due to different methods and constraints being adopted for sample selection. G93 used a combination of friends-of-friends and Materne-Tully (Materne 1978; Tully 1980) methods to identify galaxy groups based on a galaxy sample with a $B$-band magnitude cutoff of 14.0 in the Lyon-Meudon Extragalactic Database (LEDA; Paturel et al. 1988). T15 defined the galaxy group differently based on Two Micron All Sky Survey (2MASS) data and used scaling relations as additional selection parameters. There are caveats for all of these methods and selection criteria.

In Figure 7, we show the galaxy members of the NGC 1407 (grey), Eridanus (blue) and NGC 1332 (red) groups as identified in B06. The ellipses represent the maximum radial extent of the groups based on $r_{500}$ (radius that encompasses overdensity of 500 times the critical density) in Table 3 of B06. The encompassed virial masses are 7.9, 2.1 and $1.4 \times 10^{13} \mathrm{M}_{\odot}$ for NGC 1407, Eridanus and NGC 1332 groups, respectively. The locations of two detected $\mathrm{H}_{\mathrm{I}}$ clouds are shown as orange triangles. The black and red edgecolor circles represent the $\mathrm{H}$ I detected galaxies from this study. The former have been identified as group members in B06, G93 and/or T15 catalogues and the latter do not have a membership confirmation. These eight (open red circles) galaxies are relatively faint $(r>15 \mathrm{mag}$, see Table 4) and do not have 2MASS photometry. Thus, it is likely that these eight galaxies were missed in the T15 catalogue. To assess if these eight galaxies are associated with the Eridanus group, we calculate the velocity difference between each galaxy and the mean central velocity of the Eridanus group $\left(1492 \mathrm{~km} \mathrm{~s}^{-1}\right)$ and then compare it to 3 times the velocity dispersion of $228 \mathrm{~km} \mathrm{~s}^{-1}$ (refer to T15 and TCS16). With this criterion, these eight galaxies are considered as part of the Eridanus group. We also consider detected $\mathrm{H}_{\mathrm{I}}$ galaxies in either B06, G93 and/or T15 as part of their associated groups. Table 2 summarises 


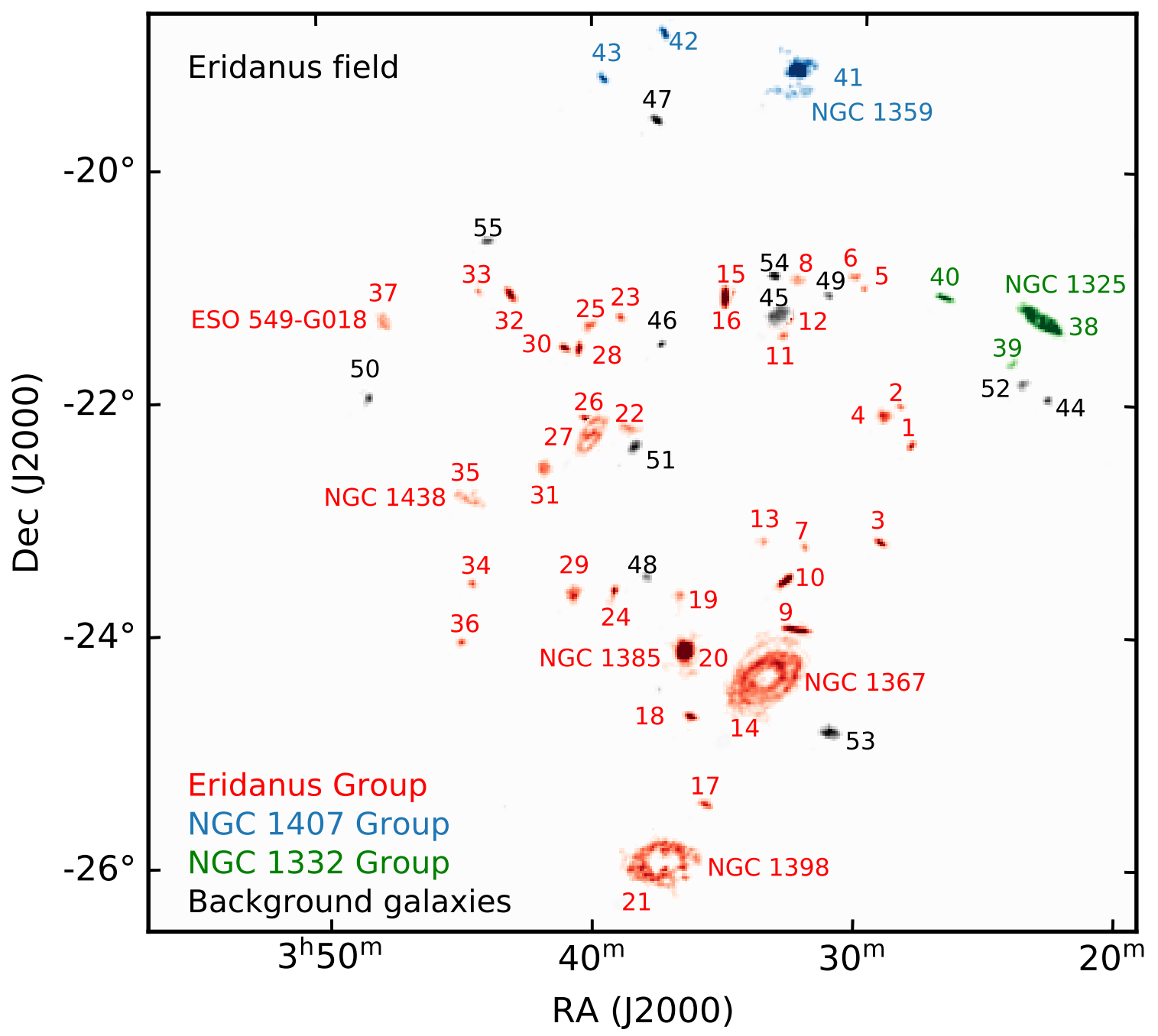

Figure 4. Integrated $\mathrm{H}_{\mathrm{I}}$ intensity map of individual sources in the Eridanus Supergroup and in the background. Sources of the Eridanus group, the NGC 1407 group, the NGC 1332 group and background galaxies are in red, blue, green and black, respectively. All sources are enlarged by a factor of 4.5 for clarity. Designated ID number for all $55 \mathrm{H}$ i detected sources are labelled.

the group membership identified in different catalogues and this study.

\section{PHYSICAL PARAMETERS}

The parameters derived in this section are given in Tables 3 and 4.

\subsection{Distance and Recession Velocity}

The recession velocity in the cosmic microwave background (CMB) reference frame, $V_{\mathrm{CMB}}$, is given by

$V_{\mathrm{CMB}}=V_{\mathrm{opt}}+V_{\text {apex }}\left[\sin b \sin b_{\text {apex }}+\cos b \cos b_{\text {apex }} \cos \left(l-l_{\text {apex }}\right)\right]$,

where $l$ and $b$ are Galactic coordinates, $l_{\text {apex }}=264.14^{\circ}, b_{\text {apex }}=$ $48.26^{\circ}$ and $V_{\text {apex }}=371.0 \mathrm{~km} \mathrm{~s}^{-1}$ (Fixsen et al. 1996). In Figure 8, we show the $V_{\mathrm{CMB}}$ distribution of $\mathrm{H}_{\mathrm{I}}$ detected galaxies in the Eridanus field. The mean $V_{\mathrm{CMB}}$ for the Eridanus supergroup is $\sim 1500 \mathrm{~km} \mathrm{~s}^{-1}$. The "wall" behind the Eridanus supergroup at $4000 \mathrm{~km} \mathrm{~s}^{-1}$ is also shown. There are fewer galaxies beyond $4000 \mathrm{~km} \mathrm{~s}^{-1}$ as a result of decreasing sensitivity of the WALLABY data toward higher redshift.

For galaxies behind the Eridanus supergroup, we use $D_{\mathrm{H}}=$ $V_{\mathrm{CMB}} / H_{0}$ and $D_{\mathrm{L}}=(1+z) D_{\mathrm{c}}$ to calculate the Hubble and luminosity distances, respectively. At low redshift $(z<0.05)$, the co-moving distance, $D_{\mathrm{c}} \approx D_{\mathrm{H}}$, hence $D_{\mathrm{L}} \approx(1+z) D_{\mathrm{H}}$.

For the Eridanus subgroups, we consider redshift-independent measurements for their distances to avoid the effects of peculiar velocities, which are significant for galaxies with recession velocities less than $2000 \mathrm{~km} \mathrm{~s}^{-1}$ (Marinoni et al. 1998). There are several redshift-independent methods for determining distances, including the use of the globular cluster luminosity function (GCLF; Richtler 2003 and references therein). The GCLF is derived from the total observed population of GCs of a galaxy. The distance modulus $(m-M)$ derived from the GCLF of NGC 1407, NGC 1332 and NGC 1395 are $31.6 \pm 0.1 \mathrm{mag}$ (Forbes et al. 2006), $31.7 \pm 0.24 \mathrm{mag}$ (Kundu \& Whitmore 2001) and $31.79 \pm 0.16$ mag (Escudero et al. 2018), respectively. These correspond to distances of $20.9 \mathrm{Mpc}, 21.9 \mathrm{Mpc}$ 


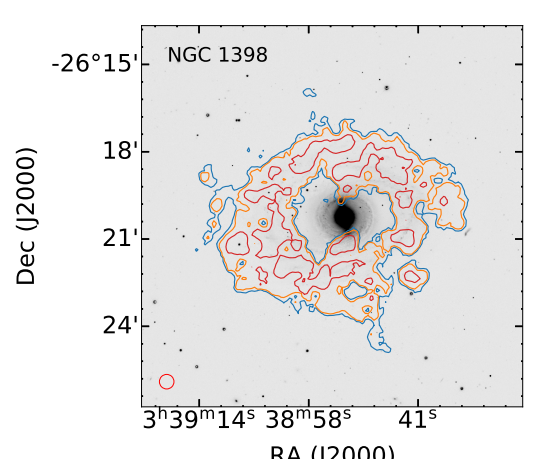

RA (J2000)

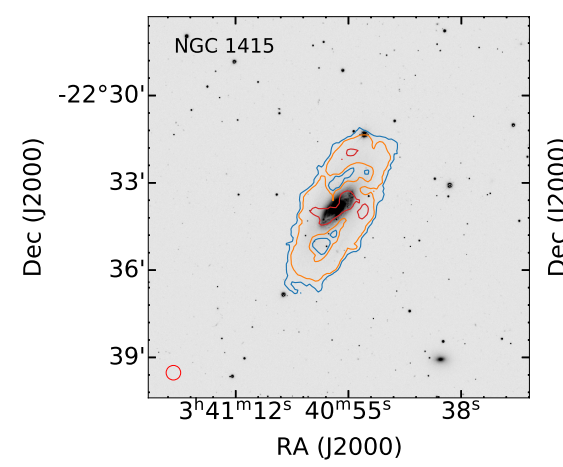

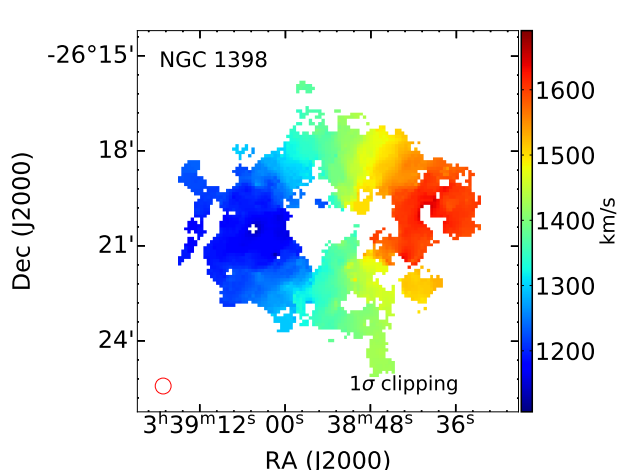

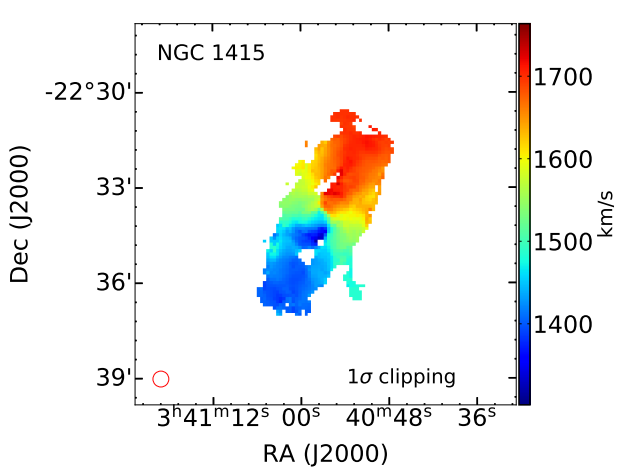

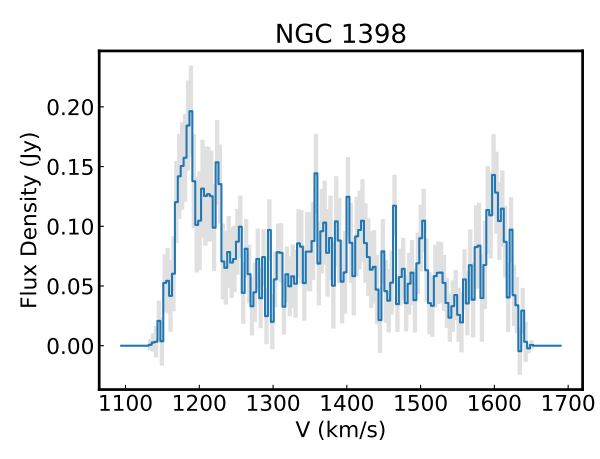

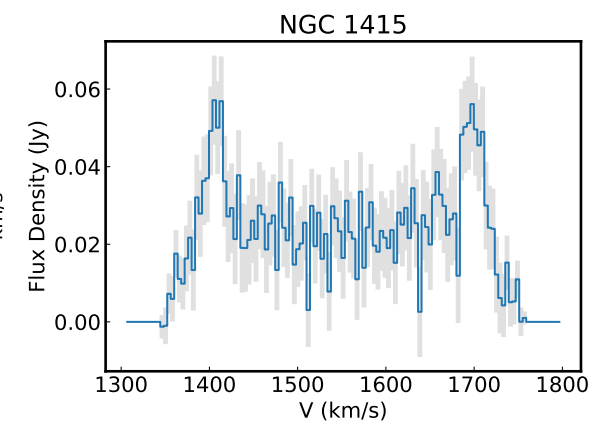

Figure 5. Examples of WALLABY H i sources. A mean correction factor of $20 \%$ has been applied to integrated $\mathrm{H}$ i column density maps, flux density and local RMS of spectra. The synthesised beam of $30^{\prime \prime} \times 30^{\prime \prime}$ is plotted at the left bottom corner of each sub-plot as a reference. Left: Integrated H i column density maps of individual sources overlaid onto the DR8 DESI Legacy Imaging Survey $g$-band stacked images. The colour scale of blue, orange, red, purple, and olive green represent the contour levels of $1,2,5,10,20 \times 10^{20} \mathrm{~cm}^{-2}$, respectively. The $3 \sigma$ of H i column density sensitivity level for a typical RMS of 1.7 mJy beam ${ }^{-1}$ over $20 \mathrm{~km} \mathrm{~s}^{-1}$ is $1.2 \times 10^{20} \mathrm{~cm}^{-2}$. Middle: 1 st moment velocity field maps of individual sources. Pixels above $n-\sigma$ are plotted and the value for $n$ is given at bottom

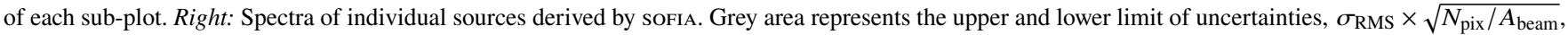
where the Gaussian beam area, $A_{\text {beam }}=\pi \theta_{\text {bmaj }} \theta_{\mathrm{bmin}} /(4 \ln 2)$. Note: This figure is published in its entirety as Supporting Information with the electronic version of the paper. A portion is shown here.

and $22.8 \mathrm{Mpc}$. We note that the distance to the Eridanus group cannot solely rely on the measurement of NGC 1395 because it is not at the dynamical/virial centre of the group. Eridanus is dynamically young and is still evolving. Considering the distances and their associated errors of the more mature subgroups (NGC 1407 and NGC 1332), we adopt a distance of $21 \mathrm{Mpc}$ for all subgroups.

\subsection{H I Linewidth}

The $\mathrm{H}_{\mathrm{I}}$ linewidth measured at 20 percent of the level of each peak $\left(w_{20}\right)$ from sofia needs to be corrected for instrumental broadening. The correction is given by

$w_{20}^{c}=\frac{\sqrt{w_{20}^{2}-(\Delta s)^{2}}}{\Delta v} \times \Delta V_{\text {rest }}$.

$w_{20}$ has a default unit of $\mathrm{Hz}$ from sofiA. The instrumental broadening, $\Delta s=2 \Delta v \lambda$, where $\lambda$ is the broadening parameter and $\Delta v$ is assumed to be equal to the observed channel width in $\mathrm{Hz}$ (Springob et al. 2005). We adopt $\lambda=0.5$ for a typical profile shape. The channel width in the source rest frame $\left(\Delta V_{\text {rest }}\right)$ in units of $\mathrm{km} \mathrm{s}^{-1}$ can be calculated using the following equation:

$\Delta V_{\text {rest }} \simeq \Delta v \frac{c(1+z)}{v_{\mathrm{HI}}}$ where $c$ is the speed of light, $z$ is redshift and $v_{\mathrm{HI}}$ is rest frequency of the $\mathrm{H}_{\mathrm{I}}$ emission line (Meyer et al. 2017). Subsequently, equation 2 can be simplified as follows,

$w_{20}^{c}=\sqrt{w_{20}^{2}-(\Delta s)^{2}} \times \frac{c(1+z)}{v_{\mathrm{HI}}}$.

\subsection{Stellar mass}

We use the existing colour-stellar mass relations of Bell et al. (2003) (hereafter B03) to derive stellar masses of our galaxies. This relies on the photometry measurements from the co-added images of the DESI Legacy Imaging Surveys DR8 (Dey et al. 2019), which are primarily based on the Dark Energy Camera Legacy Survey (DECaLS) southern observations. The straight-forward way to obtain co-added images is through the cutout tool, in which the acquired galaxy is centred and stitched if it falls between two or more CCD tiles. However, these cutout images are weighted averages of the individual images and subjected to varying point-spread-functions (PSF). To verify if the use of such co-added cutout images would result in inaccurate photometry measurements, we obtain another set of co-added images by first finding out the identification names of the bricks in which galaxies are located and then retrieving the co-added images via the 
Table 2. Group membership.

\begin{tabular}{|c|c|c|c|c|c|}
\hline Designation & $\begin{array}{c}V \\
\left(\mathrm{~km} \mathrm{~s}^{-1}\right) \\
(2)\end{array}$ & B06 & G93 & T15/TCS16 & $\begin{array}{c}\text { This Stutdy } \\
\text { (6) }\end{array}$ \\
\hline & & \multicolumn{4}{|c|}{ Group Identification } \\
\hline WALLABY J034002-192200 & 1216 & NGC 1407 & $\ldots$ & $\ldots$ & NGC 1407 \\
\hline WALLABY J033752-190024 & 1218 & NGC 1407 & $\ldots$ & $\ldots$ & NGC 1407 \\
\hline WALLABY J033019-210832 & 1226 & $\ldots$ & $\ldots$ & $\ldots$ & Eridanus \\
\hline WALLABY J033408-232125 & 1262 & $\ldots$ & $\ldots$ & $\ldots$ & Eridanus \\
\hline WALLABY J033047-210333 & 1292 & Eridanus & $\ldots$ & $\ldots$ & Eridanus \\
\hline WALLABY J033854-262013 & 1373 & X 6dF & $\ldots$ & TSK 849, Nest 200100 & Eridanus \\
\hline WALLABY J032455-214701 & 1456 & NGC 1332 & $\ldots$ & $\ldots$ & NGC 1332 \\
\hline WALLABY J033501-245556 & 1458 & X 6dF & $\ldots$ & TSK 849, Nest 200100 & Eridanus \\
\hline WALLABY J033723-235753* & 1469 & $\ldots$ & $\ldots$ & $\ldots$ & Eridanus \\
\hline WALLABY J033728-243010 & 1497 & $\mathrm{X}$ & LGG 97 & TSK 849, Nest 200100 & Eridanus \\
\hline WALLABY J033327-213352 & 1509 & Eridanus & $\ldots$ & $\ldots$ & Eridanus \\
\hline WALLABY J033527-211302 & 1518 & Eridanus & $\ldots$ & $\ldots$ & Eridanus \\
\hline WALLABY J034517-230001 & 1546 & X & LGG 97 & TSK 849, Nest 200100 & Eridanus \\
\hline WALLABY J034056-223350 & 1552 & Eridanus & $\ldots$ & TSK 849, Nest 200100 & Eridanus \\
\hline WALLABY J034219-224520 & 1569 & Eridanus & $\ldots$ & $\ldots$ & Eridanus \\
\hline WALLABY J034434-211123 & 1578 & $\ldots$ & $\ldots$ & $\ldots$ & Eridanus \\
\hline WALLABY J034814-212824 & 1586 & X 6dF & LGG 97 & TSK 849 & Eridanus \\
\hline WALLABY J032425-213233 & 1588 & NGC 1332 & $\ldots$ & TSK 849 & NGC 1322 \\
\hline WALLABY J033617-253615 & 1590 & X 6dF & $\ldots$ & Nest 200100 & Eridanus \\
\hline WALLABY J034337-211418 & 1612 & Eridanus & $\ldots$ & $\ldots$ & Eridanus \\
\hline WALLABY J033941-235054 & 1622 & Eridanus & $\ldots$ & $\ldots$ & Eridanus \\
\hline WALLABY J033921-212450 & 1622 & Eridanus & $\ldots$ & $\ldots$ & Eridanus \\
\hline WALLABY J032900-220851 & 1627 & $\mathrm{X}$ & LGG 97 & $\ldots$ & Eridanus \\
\hline WALLABY J034131-214051 & 1644 & Eridanus & $\ldots$ & $\ldots$ & Eridanus \\
\hline WALLABY J034036-213129 & 1644 & Eridanus & $\ldots$ & $\ldots$ & Eridanus \\
\hline WALLABY J032937-232103 & 1657 & X 6dF & $\ldots$ & $\ldots$ & Eridanus \\
\hline WALLABY J033257-210513 & 1665 & Eridanus & $\ldots$ & $\ldots$ & Eridanus \\
\hline WALLABY J032735-211339 & 1686 & NGC 1332 & $\ldots$ & $\ldots$ & NGC 1332 \\
\hline WALLABY J034057-214245 & 1695 & Eridanus & $\ldots$ & $\ldots$ & Eridanus \\
\hline WALLABY J034522-241208 & 1733 & $\ldots$ & $\ldots$ & $\ldots$ & Eridanus \\
\hline WALLABY J032941-221642 & 1755 & $\mathrm{X} 6 \mathrm{dF}$ & LGG 97 & $\ldots$ & Eridanus \\
\hline WALLABY J033228-232245 & 1755 & $\mathrm{X}$ & $\ldots$ & $\ldots$ & Eridanus \\
\hline WALLABY J034040-221711 & 1774 & Eridanus & $\ldots$ & $\ldots$ & Eridanus \\
\hline WALLABY J032831-222957 & 1774 & $\ldots$ & $\ldots$ & $\ldots$ & Eridanus \\
\hline WALLABY J033537-211742 & 1802 & Eridanus & $\ldots$ & $\ldots$ & Eridanus \\
\hline WALLABY J033326-234246 & 1810 & X & LGG 97 & Nest 200100 & Eridanus \\
\hline WALLABY J034456-234158 & 1819 & $\mathrm{X}$ & $\ldots$ & $\ldots$ & Eridanus \\
\hline WALLABY J033653-245445 & 1842 & $\mathrm{X}$ & $\ldots$ & Nest 200100 & Eridanus \\
\hline WALLABY J033341-212844 & 1859 & Eridanus & $\ldots$ & $\ldots$ & Eridanus \\
\hline WALLABY J033911-222322* & 1879 & $\ldots$ & $\ldots$ & $\ldots$ & Eridanus \\
\hline WALLABY J034114-235017 & 1885 & Eridanus & $\ldots$ & Nest 200100 & Eridanus \\
\hline WALLABY J033302-240756 & 1915 & X 6dF & $\ldots$ & Nest 200100 & Eridanus \\
\hline WALLABY J033347-192946 & 1964 & $\mathrm{X} 6 \mathrm{dF}$ & LGG 100 & TSK 863 & NGC 1407 \\
\hline
\end{tabular}

Cols (1)-(2): Designation and H i spectral line derived velocity - cz. Col (3): Identification in B06 - member of Eridanus, NGC 1332 or NGC 1407 group; X represents galaxies that are not identified as any member of the groups but with $6 \mathrm{dF}$ identification. $\mathrm{X} 6 \mathrm{dF}$ indicates galaxies identified in NED but not observed in the $6 \mathrm{dF}$ survey. Col (4): Identification in G93 - member of LGG 97 or LGG 100. Col (5): Identification in T15 or TCS16 - member of TSK 849, TSK 863 and/or Nest 200100. Col (6): Identification in this study. *: H I clouds.

NERSC server ${ }^{5}$. By comparing five fluxes obtained from cutout and non-cutout (non-stitched) co-added images, we find that the difference is small and within $0.02 \mathrm{mag}$. This implies that the issue with varying PSF is minor and we decide to use the cutout co-added im-

\footnotetext{
5 Available at https://portal.nersc.gov/project/cosmo/data/ legacysurvey/dr8/south/coadd/
}

ages for the measurement to bypass the effort of stitching non-cutout co-added images manually.

We perform the $g$ and $r$-band photometry measurement using PROFOUND (Robotham et al. 2018). PROFOUND is used in rather than the traditional circular or ellipse based method because it is useful for measuring fluxes of low surface brightness (LSB) galaxies, detecting faint signal with high noise level background and isolating foreground/background objects. Our sample consists faint dwarfs and LSB galaxies. To identify background objects, we use the 3-colour 


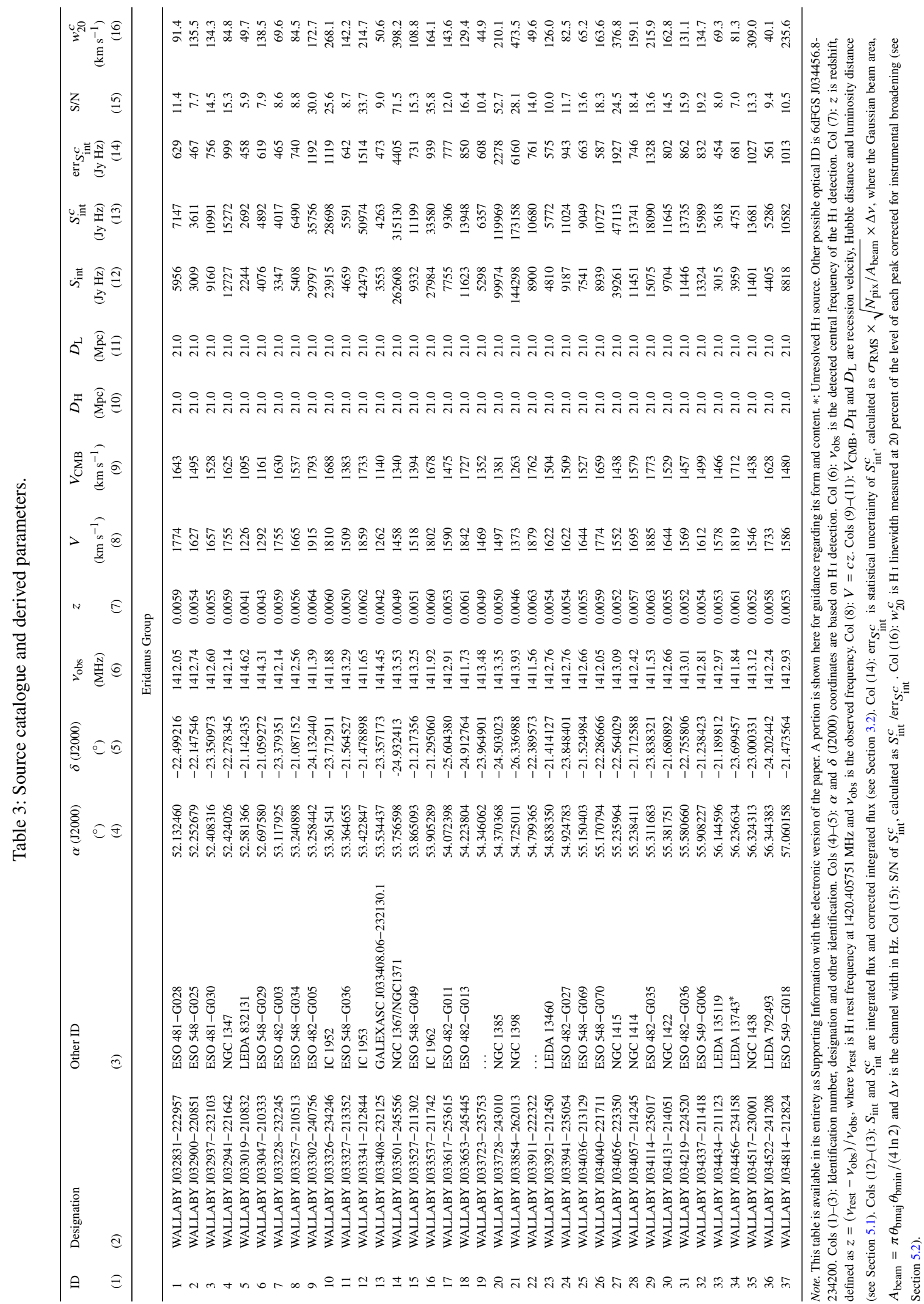



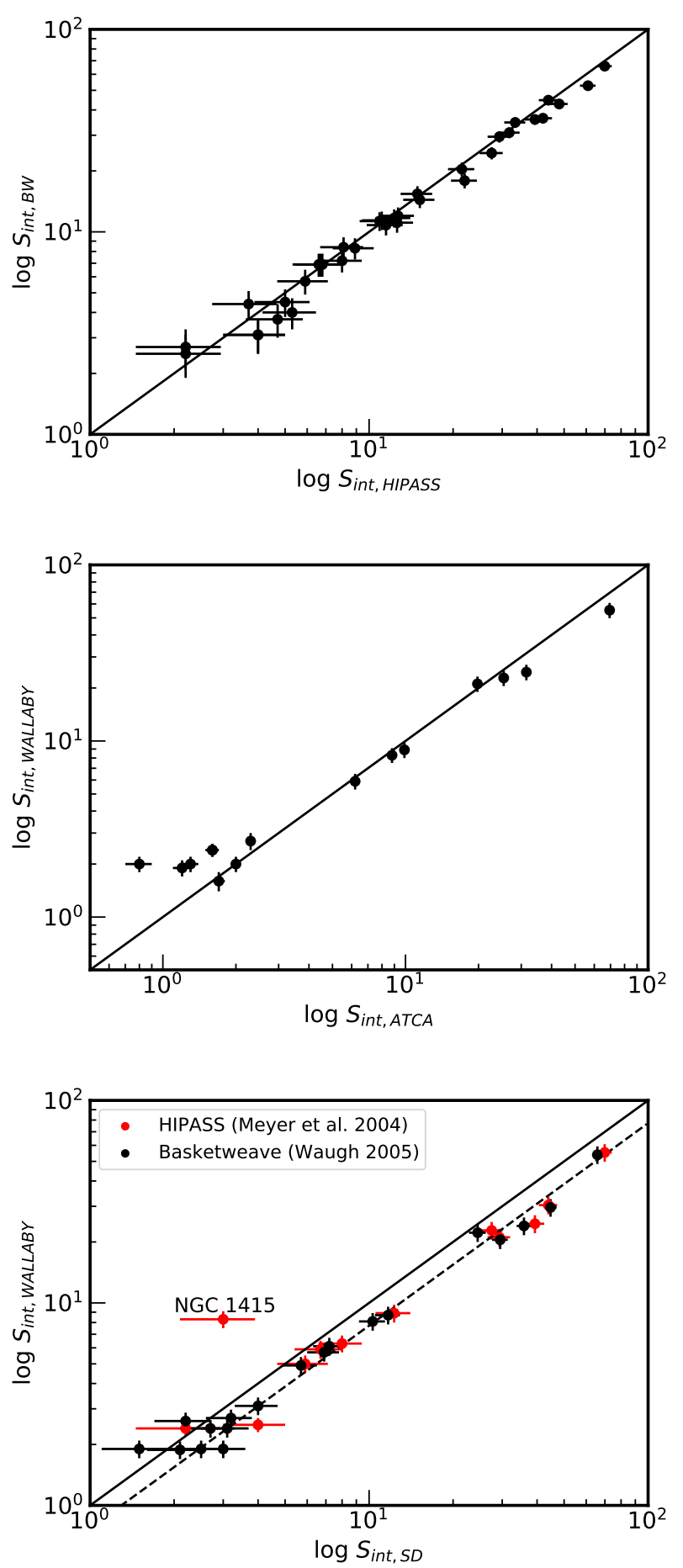

Figure 6. Comparison of $S_{\text {int }}$ between various studies. The solid line is the $1: 1$ unity line. We adopt $10 \%$ of $S_{\text {int }}$ as errors for WALLABY data points as the statistical uncertainties as listed in Table 3 do not take into account calibration uncertainties. Top: Comparison of $S_{\text {int }}$ between BW (Waugh 2005) and HIPASS (Meyer et al. 2004). Middle: Comparison of $S_{\text {int }}$ between WALLABY pre-pilot and ATCA (Waugh 2005). Bottom: Comparison of $S_{\text {int }}$ between WALLABY pre-pilot and single-dish data. The black and red dots represent the $S_{\text {int }}$ from BW and HIPASS, respectively. The dashed line is the fitted line, indicating a $\sim 20 \%$ systematic offset between the WALLABY and single-dish data.

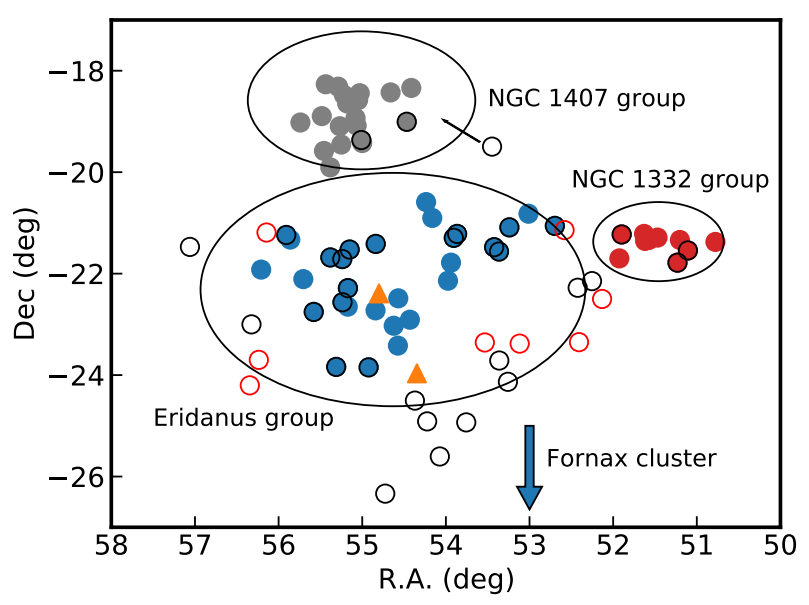

Figure 7. Same as Figure 1 except adding detected H I sources in this study. Both red and black edge colour circles (including those filled) represent the detected $\mathrm{H}_{\mathrm{I}}$ galaxies. Two H i clouds are shown in orange triangles.

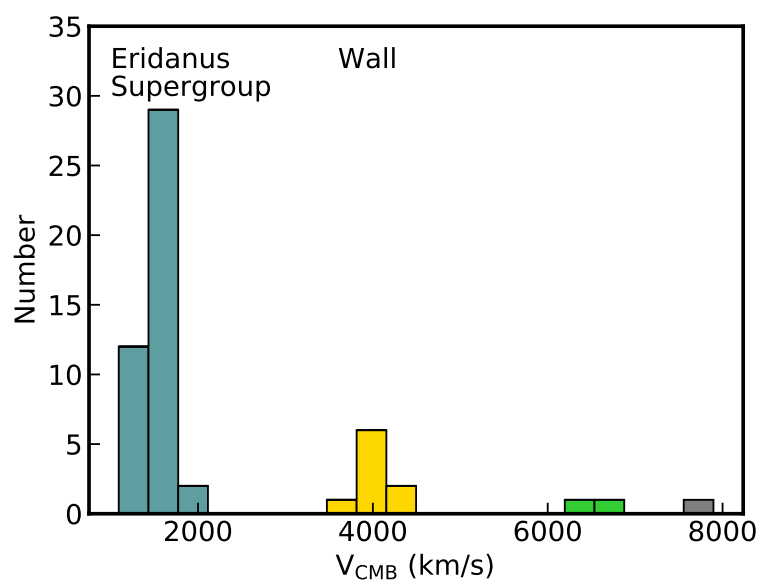

Figure 8. Histogram of recession velocities $\left(V_{\mathrm{CMB}}\right)$. Different colours represent $\mathrm{H}_{\mathrm{I}}$ detections in different groups of velocity.

composite images for guidance. The background galaxies tend to be redder in colour. We also cross-check the PROFOUND photometry measurements of a few bright galaxies with the DR8 tractor catalogue and find them to be consistent within $0.02 \mathrm{mag}$.

To calculate the extinction corrected (intrinsic) magnitude, we adopt the wavelength-dependent extinction law that is parametrised by $R_{V}=A_{V} / E(B-V)$ (Cardelli et al. 1989). Using the reddening law in Fitzpatrick (1999) and with $R_{V}=3.1$, the tabulated $A_{\lambda}$ values for Dark Energy Survey (DES) $g$ and $r$-bands are $A_{g}=3.237 E(B-V)$ and $A_{r}=2.176 E(B-V)$, respectively (see Table 6 of Schlafly \& Finkbeiner 2011). We estimate the Galactic dust extinction, $E(B-V)$, by using the re-calibrated SFD all-sky extinction maps (Schlegel et al. 1998; Schlafly \& Finkbeiner 2011). The intrinsic magnitude is then calculated as $\operatorname{mag}_{0}=\operatorname{mag}-A_{\lambda}$. Subsequently, we obtain the stellar masses by employing the mass-to-light ratio $(M / L)$ relation in $\mathrm{B} 03$ as follow:

$\log \left(\frac{M_{*}}{L_{r}}\right)=-0.306+1.097(g-r)$ 
where $L_{r}$ (luminosity) $=M_{r \text {, abs }}-M_{\text {sun, abs }}$ in $L_{\odot}$. The absolute magnitude of the Sun ( $M_{\text {sun, abs }}$ ) of different DES wavebands is given in Willmer (2018) and the $D_{\mathrm{L}}$ in Table 3 is used to calculate the $M_{r \text {,abs }}$. This $M / L$ relation adopts the "diet" Salpeter (1955) initial mass function (IMF) that contains only $70 \%$ of the mass due to a lower number of faint low-mass stars in their samples. To use the Chabrier (2003) IMF, the $M_{*}$ values need to be scaled by 0.61 according to Madau \& Dickinson (2014). This is to put our data points on the same scale with other surveys for comparison in Section 6.

We find that stellar masses for galaxies in the Eridanus supergroup and background galaxies range from $1.2 \times 10^{6}$ to $9.5 \times 10^{10} \mathrm{M}_{\odot}$ and $6.3 \times 10^{7}$ to $2.1 \times 10^{10} \mathrm{M}_{\odot}$, respectively. By comparing the scaled stellar masses with those calculated with the $M / L$-colour relations of Zibetti et al. (2009) (hereafter Z09), we find that the difference is relatively small with an average \pm 0.02 dex for all galaxies. The galaxies with high stellar mass $\left(M_{*} \gtrsim 10^{10} \mathrm{M}_{\odot}\right)$ tend to have lower derived $M_{*}$ when using the colour-stellar mass relations of B03. Using the colour-stellar mass relations of Z09, the lowest mass galaxy (WALLABY J033408-232125) in this study is 0.32 dex smaller in stellar mass. The differences are in part due to different stellar population synthesis models being adopted in the two studies (see Z09 for a detailed discussion). We adopt the stellar masses derived from B03. We do not find that the differences alter our conclusions.

\subsection{HI mass and HI-deficiency parameter}

Assuming that the H i sources are optically thin, we can calculate the H I mass using the following equation:

$\left(\frac{M_{\mathrm{HI}}}{\mathrm{M}_{\odot}}\right) \simeq 49.7\left(\frac{D_{\mathrm{L}}}{\mathrm{Mpc}}\right)^{2}\left(\frac{S_{\text {int }}}{\mathrm{Jy} \mathrm{Hz}_{\mathrm{Hz}}}\right)$,

where $D_{\mathrm{L}}$ is the luminosity distance and $S_{\text {int }}$ is the integrated flux (Meyer et al. 2017). In Figure 9, we show the histogram of the HI masses of the detected galaxies and HI clouds in the Eridanus group (blue and light blue), galaxies in the NGC 1407 and NGC 1332 groups (orange and green), and background galaxies (grey). The background galaxies have a $\mathrm{H}_{\mathrm{I}}$ mass distribution of $\sim 10^{8.9-10} \mathrm{M}_{\odot}$. The $\mathrm{H}_{\mathrm{I}}$ mass of the Eridanus supergroup covers a range of $10^{7.7-9.8} \mathrm{M}_{\odot}$ and has a median value of $10^{8.3} \mathrm{M}_{\odot}$. We have recovered a significant number of galaxies with $\mathrm{H}_{\mathrm{I}}$ masses lower than those found in Omar \& Dwarakanath (2005b), where their derived $\mathrm{H}_{\mathrm{I}}$ mass ranges from $\sim 10^{8.3}$ to $10^{9.9} \mathrm{M}_{\odot}$.

To quantify the relative $\mathrm{H}$ I content of galaxies, we use the $\mathrm{H}$ I deficiency parameter, $\mathrm{DEF}_{\mathrm{HI}}$. It is defined as the logarithmic difference between the expected and observed $\mathrm{H}_{\mathrm{I}}$ masses of a galaxy,

$\mathrm{DEF}_{\mathrm{HI}}=\log \left(M_{\mathrm{HI}, \exp } / \mathrm{M}_{\odot}\right)-\log \left(M_{\mathrm{HI}, \mathrm{obs}} / \mathrm{M}_{\odot}\right)$

(Haynes \& Giovanelli 1984). The expected H I mass of each galaxy can be determined by its morphology and size (Haynes \& Giovanelli 1984; Solanes et al. 1996; Jones et al. 2018) or magnitude based H I scaling relations (Dénes et al. 2014; hereafter D14). The morphology and size method is subject to inhomogenous morphological classification and mostly limited to bright galaxies with known classification from previous studies. We have explored this method and find it unsuitable for our study given that there is a large number of new LSB galaxies and dwarfs without a homogenous morphological classification. Thus, we adopt the magnitude based $\mathrm{H}_{\text {I }}$ scaling relation to determine the expected $\mathrm{H}_{\mathrm{I}}$ mass, which is given by

$\log \left(M_{\mathrm{HI}, \exp } / \mathrm{M}_{\odot}\right)=3.43-0.3 \times M_{r}$

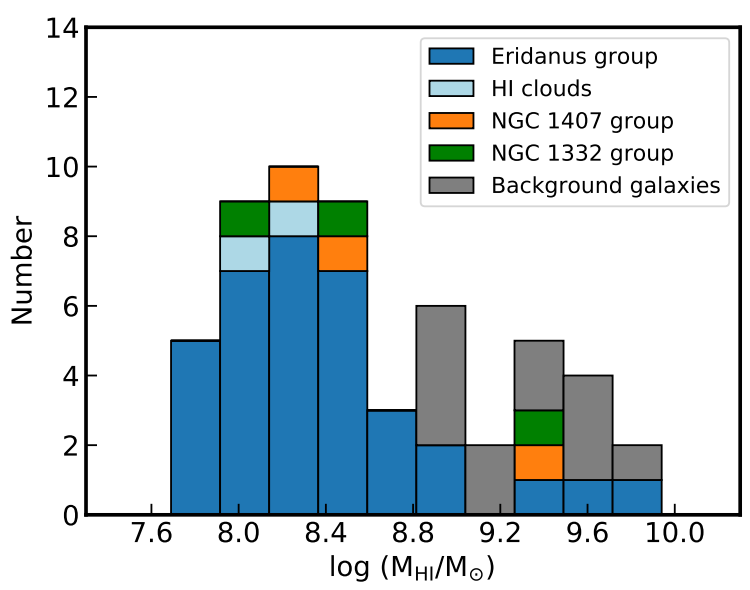

Figure 9. Histogram of $\mathrm{H}_{\mathrm{I}}$ masses on a logarithmic scale. Galaxies and $\mathrm{H}_{\mathrm{I}}$ clouds in the Eridanus group are represented in blue and light blue. Galaxies in the NGC 1407 group, the NGC 1332 group and background are also represented in orange, green and grey, respectively.

where $M_{r}$ is the absolute magnitude in SDSS $r$-band ${ }^{6}$ (see Table 3 of D14). We consider a galaxy to be $\mathrm{H}_{\mathrm{I}}$ deficient if $\mathrm{DEF}_{\mathrm{HI}}>+0.3$ and $\mathrm{H}_{\mathrm{I}}$ excess if $\mathrm{DEF}_{\mathrm{HI}}<-0.3$. These criteria correspond to less than half or more than twice $\mathrm{H}_{\mathrm{I}}$ mass on average than expected. The histogram of $\mathrm{DEF}_{\mathrm{HI}}$ for all galaxies is shown in Figure 10. 20 galaxies are considered to be $\mathrm{H}_{\mathrm{I}}$ deficient and one $\mathrm{H}_{\mathrm{I}}$ excess in the Eridanus group. There is one $\mathrm{H}$ i deficient galaxy within our limited sample of the NGC 1407 group. There are also four background galaxies considered to be $\mathrm{H}$ i excess, and with one galaxy considered to be $\mathrm{H}_{\mathrm{I}}$ deficient.

The on-sky distribution of $\mathrm{DEF}_{\mathrm{HI}}$ of galaxies in the Eridanus supergroup is shown in Figure 11, where the majority of $\mathrm{H}_{\mathrm{I}}$ deficient galaxies are in close proximity with other $\mathrm{H}$ i detected galaxies. We note that the empirical scaling relation has a large instrinsic scatter with stellar luminosity alone as a prediction of $\mathrm{H}_{\mathrm{I}}$ mass. A more physically motivated derivation of the $\mathrm{H}_{\mathrm{I}}$ deficiency parameter has been explored using the stellar mass and angular momentum (see Obreschkow et al. 2016; Li et al. 2020). This method is adopted and the interpretation of $\mathrm{H}_{\mathrm{I}}$ deficiency of galaxies is further discussed in Murugeshan et al. (2021).

Overall, the Eridanus group consists of more $\mathrm{H}$ i deficient galaxies than other Local Volume galaxy groups (see e.g. Reynolds et al. 2019). We also find that the H I deficiency parameter does not show any correlation as a function of projected distance from the Eridanus group centre. This is consistent with the study of $\mathrm{H}_{\mathrm{I}}$ galaxies in 16 loose groups, where $\mathrm{H}_{\mathrm{I}}$ deficient galaxies are not necessarily found at the centres of groups but within $\sim 1 \mathrm{Mpc}$ from the group centre (Kilborn et al. 2009). The non-correlation suggests that the pre-processing in the Eridanus group is yet to reach a stable stage of evolution. This is somewhat expected for a dynamically young system that is in the process of merging.

${ }^{6}$ SDSS $r$-band is similar to the $r$-band in DECaLS (Dey et al. 2019). 


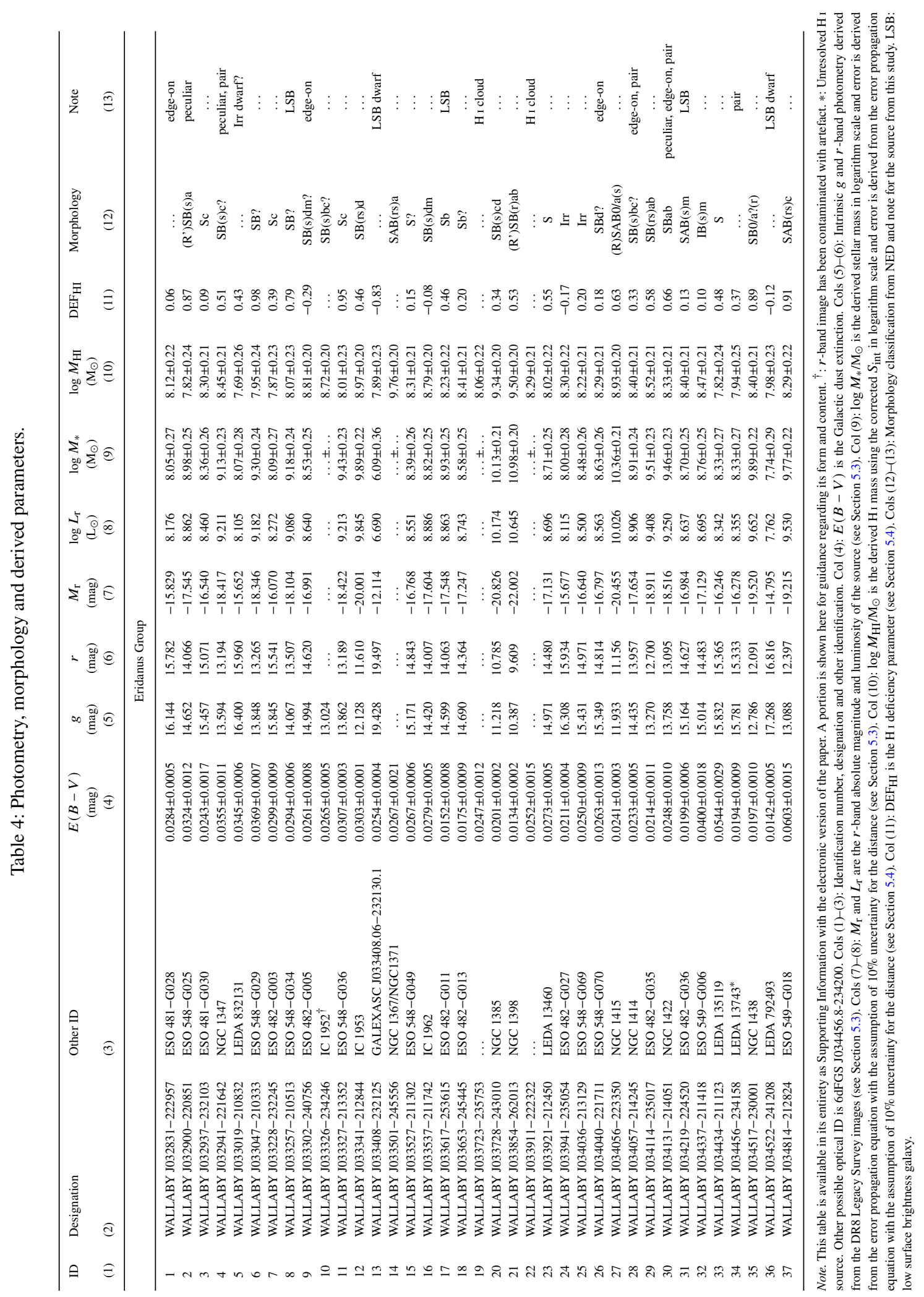




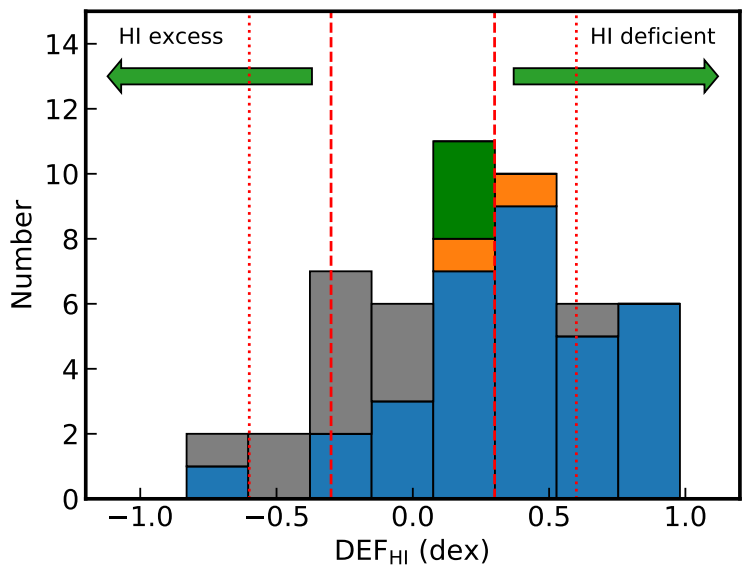

Figure 10. Same as Figure 9 except on the distribution of H I deficiency parameter. The red dashed lines mark the boundaries of galaxies with normal $\mathrm{H}_{\text {I }}$ content $\left(-0.3<\mathrm{DEF}_{\mathrm{HI}}<+0.3\right)$ with respect to the expected $\mathrm{H}_{\mathrm{I}}$ from the scaling relation of Dénes et al. (2014). Galaxies with $\mathrm{DEF}_{\mathrm{HI}}>+0.3$ and $<$ -0.3 are considered $\mathrm{H}_{\mathrm{I}}$ deficient and $\mathrm{H}_{\mathrm{I}}$ excess, respectively. The red dotted lines indicate the conservative definition of $\mathrm{H}_{\mathrm{I}}$ deficient and $\mathrm{H}$ I excess, i.e. $\mathrm{DEF}_{\mathrm{HI}}>+0.6$, and $\mathrm{DEF}_{\mathrm{HI}}<-0.6$.

\section{ATOMIC GAS FRACTION-STELLAR MASS SCALING RELATION}

The atomic gas fraction $\left(M_{\mathrm{HI}} / M_{*}\right)$ versus stellar mass scaling relation is a useful tool in investigating the influence of different environments on galaxy properties and $\mathrm{H}$ I content. In Figure 12, we show the atomic gas fraction as a function of stellar mass. The top left shows xGASS H I detected sample (grey circles; Catinella et al. 2018), ALFALFA.40 samples (blue and red squares; Maddox et al. 2015) and samples from this study (orange and black circles). xGASS is a stellar mass selected $\mathrm{H}_{\mathrm{I}}$ survey and its sample is limited to $9<\log \left(M_{*} / \mathrm{M}_{\odot}\right)$ $<11.5$ and $0.01<z<0.05$. The xGASS sample shows a clear trend of linear relation of decreasing $\log M_{\mathrm{HI}} / M_{*}$ with increasing $\log M_{*}$. The blue and red squares represent the atomic gas fraction (median $\log M_{\mathrm{HI}}-\log M_{*}$ ) of ALFALFA.40 samples with stellar masses derived from SDSS spectra and photometry, respectively (see tabulated values in Table 1 of Maddox et al. 2015). These empirical relations show that the gas fraction follows the same trend as in the study of xGASS at higher stellar mass $\left(>10^{9} \mathrm{M}_{\odot}\right)$ but flatten out at lower stellar mass indicating relatively higher gas content in this regime. Deviation at the lower stellar mass end is seen between the two SDSS samples. The transition to this plateau presumably traces a real change in galaxy population as galaxies with high gas content $\left(M_{\mathrm{HI}} / M_{*} \sim 100\right)$ would have been detected by ALFALFA. 40 .

Our data for the Eridanus supergroup and background galaxies are represented in orange and black circles, respectively. We find that galaxies in the Eridanus supergroup tend to have a lower atomic gas fraction for a given stellar mass than our background or xGASS or Maddox's galaxies. In top right panel of Figure 12, we show the trend being consistent with Local Volume H I Survey (LVHIS; Koribalski et al. 2018), Virgo cluster only Herschel Reference Survey (HRS; Boselli et al. 2014), The H I Nearby Galaxy Survey (THINGS; Walter et al. 2008) and Faint Irregular Galaxy Survey (FIGGS; Begum et al. 2008) samples presented in Wang et al. $(2017)^{7}$ (hereafter W17).

\footnotetext{
7 Chabrier IMF and $H_{0}=70 \mathrm{~km} \mathrm{~s}^{-1} \mathrm{Mpc}^{-1}$ were adopted.
}

The dashed line is a robust median linear fit of the data in W17, $\log \left(M_{\mathrm{HI}} / M_{*}\right)=-0.63 \times \log M_{*}+5.07$, and it is used as a guide for the trend only. As stated in W17, such lower atomic gas fraction trend is a result of the selection effect and is not caused by the use of interferometer versus single-dish data. We also rule out the use of different colour-stellar mass relations and errors of stellar/H i masses as the cause of this trend for galaxies in the Eridanus supergroup. The shift in trend toward lower atomic gas fraction is not caused by the closer vs further distance of the sample either, as can be supported by the following test. If the bias is due to the $\mathrm{H}$ i detection limit, we expect the data points of Eridanus supergroup galaxies to be shifted upward once they are placed in the same distance as background galaxies. To achieve this, we assume a distance of $52 \mathrm{Mpc}$ (comparable to the distance of our background galaxies) and calculate their $\mathrm{S} / \mathrm{N}$ of $S_{\text {int }}$. We consider those with $\mathrm{S} / \mathrm{N}$ of $S_{\text {int }} \geq 5.9$ to be detectable at this assumed distance (see bottom left panel). This value is the lowest detectable $\mathrm{S} / \mathrm{N}$ of $S_{\text {int }}$ of our galaxies sample (see Table 3). We find that the lower gas fraction trend remains when comparing to our background galaxies. This indicates that environmental effects might be at play for the galaxies in the Eridanus supergroup. It would be interesting to re-visit the $\mathrm{H}_{\mathrm{I}}$ scaling relation with the full WALLABY survey using group, void and cluster samples to probe the environmental effects in the future.

We also investigate the trend in the low mass regime by overplotting the gas-rich Local Volume dwarf samples selected from ALFALFA.40 (Huang et al. 2012; green circles), ultra-faint dwarf satellites of the Milky Way studied by Westmeier et al. (2015) (blue circles), sample from the Survey of H I in Extremely Low-mass Dwarfs (SHIELD; McQuinn et al. 2021) (yellow triangles), "almost dark" galaxies, Leo T and Leo P (Adams \& Oosterloo 2018; Ryan-Weber et al. 2008; McQuinn et al. 2015) (green and red crosses) onto the scaling relation as shown in the bottom right panel of Figure 12. Sample of Huang et al. (2012) is a subset of ALFALFA.40, which stellar masses etc of $\mathrm{H}$ I selected dwarf galaxies are re-derived via the spectral energy distribution (SED) fitting. The selection eliminates the bias of ALFALFA. 40 sample that tends to under estimate the stellar masses of low mass galaxies with the SDSS reduction pipeline. This bias contributes to a higher gas fraction and the flattening trend at the low mass end as seen in Maddox et al. (2015). The SHIELD dwarf galaxies sample $\left(M_{\mathrm{HI}} \lesssim 10^{7.2} \mathrm{M}_{\odot}\right)$ is a volumetrically complete $\mathrm{H}_{\text {I }}$ selected sample from ALFALFA. The overall gas fraction as a sample group is slightly lower than Huang's but within the scatter. We note that the SHIELD sample mostly consists of isolated dwarf galaxies.

As for the ultra-faint dwarf samples, the gas fraction being presented here are their upper limit. It is known that dwarf satellites located within the Local Group are Hi deficient (Grcevich \& Putman 2009; Putman et al. 2021). The loss of gas in these satellites is hypothesised to be the result of tidal interaction and ram-pressure stripping during perigalactic passages. The plot shows that the gas fraction of the dwarfs in the Local Group does not follow the general trend of the Local Volume $\left(M_{*}<10^{6} \mathrm{M}_{\odot}\right)$, although a future study is needed with a larger sample. It is also interesting to note that both Leo T (adopt $M_{\mathrm{HI}}$ of Leo T in Ryan-Weber et al. 2008) and Leo P appear to be outliers and are located closer to the ultra-faint dwarf samples on the guided $\mathrm{H}_{\mathrm{I}}$ scaling relation. If we adopt the $M_{\mathrm{HI}}$ of Leo $\mathrm{T}$ in the latest study of Adams \& Oosterloo (2018), the data point falls onto the guided $\mathrm{H}_{\mathrm{I}}$ scaling relation. This is the result of a significant amount of faint $\mathrm{H}$ I emission being recovered with deep $\mathrm{H}$ I observations. It is unclear if this would be the case for Leo P. 


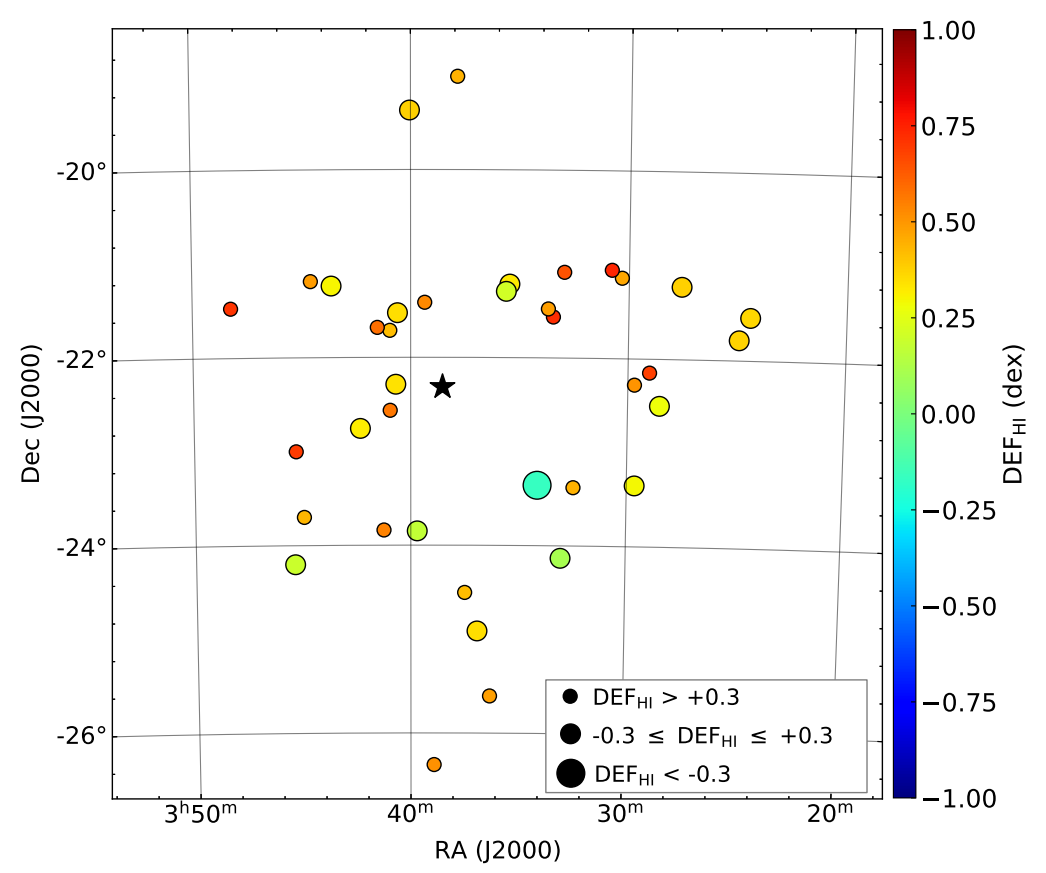

Figure 11. On-sky distribution of $\mathrm{H}_{\mathrm{I}}$ deficiency parameters of galaxies in the Eridanus supergroup. Colour and size correpond to their $\mathrm{DEF}_{\mathrm{HI}}$. The Eridanus group centre is marked with the star.

\section{STAR FORMATION RATE}

To derive the star formation rate (SFR), we use the W4 band of the neoWISE resolution enhanced mosaics (HiRes; Masci \& Fowler 2009), far-ultraviolet (FUV) and near-ultraviolet (NUV) images of the Galaxy Evolution Explorer (GALEX; Martin et al. 2005) ${ }^{8}$. We follow the method as described in W17 to perform the photometric measurements of these images for our galaxies. Subsequently, we calculate the total SFR as follows:

$\mathrm{SFR}\left(\mathrm{M}_{\odot} \mathrm{yr}^{-1}\right)=\mathrm{SFR}_{\mathrm{FUV} / \mathrm{NUV}}+\mathrm{SFR}_{\mathrm{W} 4}$,

where the SFR of each band is calculated using the model-based luminosity-to-SFR calibrations in Calzetti (2013). We adopt a fixed $D_{\mathrm{L}}$ of $21 \mathrm{Mpc}$ for the Eridanus, NGC 1407 and NGC 1332 groups and $D_{\mathrm{L}}$ for the background galaxies (see Table 3). The FUV/NUV and W4 luminosities indicate the dust unattenuated and attenuated part of the SFR, respectively. The FUV luminosities are preferred over the NUV in the estimates of SFR to minimize contamination from old stars, but when the FUV images are unavailable we use the NUV luminosities instead. We note that all galaxies in our sample have FUV detections if they have FUV observations. For galaxies that are not detected in the W4 band (mostly low-mass galaxies), the dust attenuated part of the SFR is set to zero. If there is no FUV/NUV detection, the SFR is considered to be the lower limit.

We investigate if our galaxies lie on the star-forming mainsequence (SFMS). The SFMS is a relation on the SFR- $M_{*}$ plane. Galaxies that lie on this SFMS relation are actively forming stars, are quenched if they lie below and are starbursting if they lie above. In Figure 13, we show the fitted SFR- $M_{*}$ relations derived from a sample of star forming galaxies in xCOLD GASS (Saintonge et al. 2017; hereafter S17) (red line), LSB galaxies (McGaugh et al. 2017;

\footnotetext{
${ }^{8}$ GALEX images are retrieved from the Mikulski Archive Space Telescope
} (MAST). hereafter MG17) (black line) and extremely low mass dwarfs (McQuinn et al. 2021; hereafter MQ21) (green line). The xCOLD GASS data with $\mathrm{CO}(1-0)$ (grey dots) represent the SFMS relation derived for high mass galaxies. The LSB galaxies data (blue dots) are at the low mass end. The study of MG17 shows a steep slope for the SFMS using the low mass samples but a flatter slope with the high mass samples, which is similar to the relation derived with the XCOLD GASS samples. A shallower SFMS relation slope for a similar stellar mass range is seen in MQ21's study as compared to the MG17's study. This shallower SFMS relation is consistent with the SFMS relation derived from spiral galaxies. However, with a lower birth rate parameter, MQ21 finds that a subset of the SHIELD sample galaxies would fall onto the steeper SMFS of MG17. This suggests that these subset of galaxies possess lower recent star formation that is similar to the star formation properties of LSB galaxies.

We also overplot Leo T (green cross), Leo P (red cross), our galaxies in the Eridanus supergroup (orange dots) and background galaxies (black dots) onto the SFR- $M_{*}$ plane for comparison. Our lower mass $\left(<10^{9} \mathrm{M}_{\odot}\right)$ Eridanus sample lies on the SFMS of MG17's study. Among the more massive galaxies $\left(>10^{9} \mathrm{M}_{\odot}\right)$, three of them (ESO 482-G035, NGC 1422 and ESO 548-G021) lie below the S17 SFMS relation but on MG17's. The first two are $\mathrm{H}_{\mathrm{I}}$ deficient within the Eridanus group. ESO 548-G021 is H I normal within the NGC 1332 group. It is unclear if these galaxies are quenching due to them being located in the overlapping region of these two SFMS relations. The background galaxies are in general following the SFMS relation of S17 with one (LEDA 798516) slightly above MG17's but it is still within the scatter. LEDA 798516 is possibly interacting with surrounding galaxies and this could result in bursting of star formation. Leo T and Leo P fall onto the extended relation of MG17's while the lowest stellar mass dwarf in our sample lies above it but closer to MQ21's. 

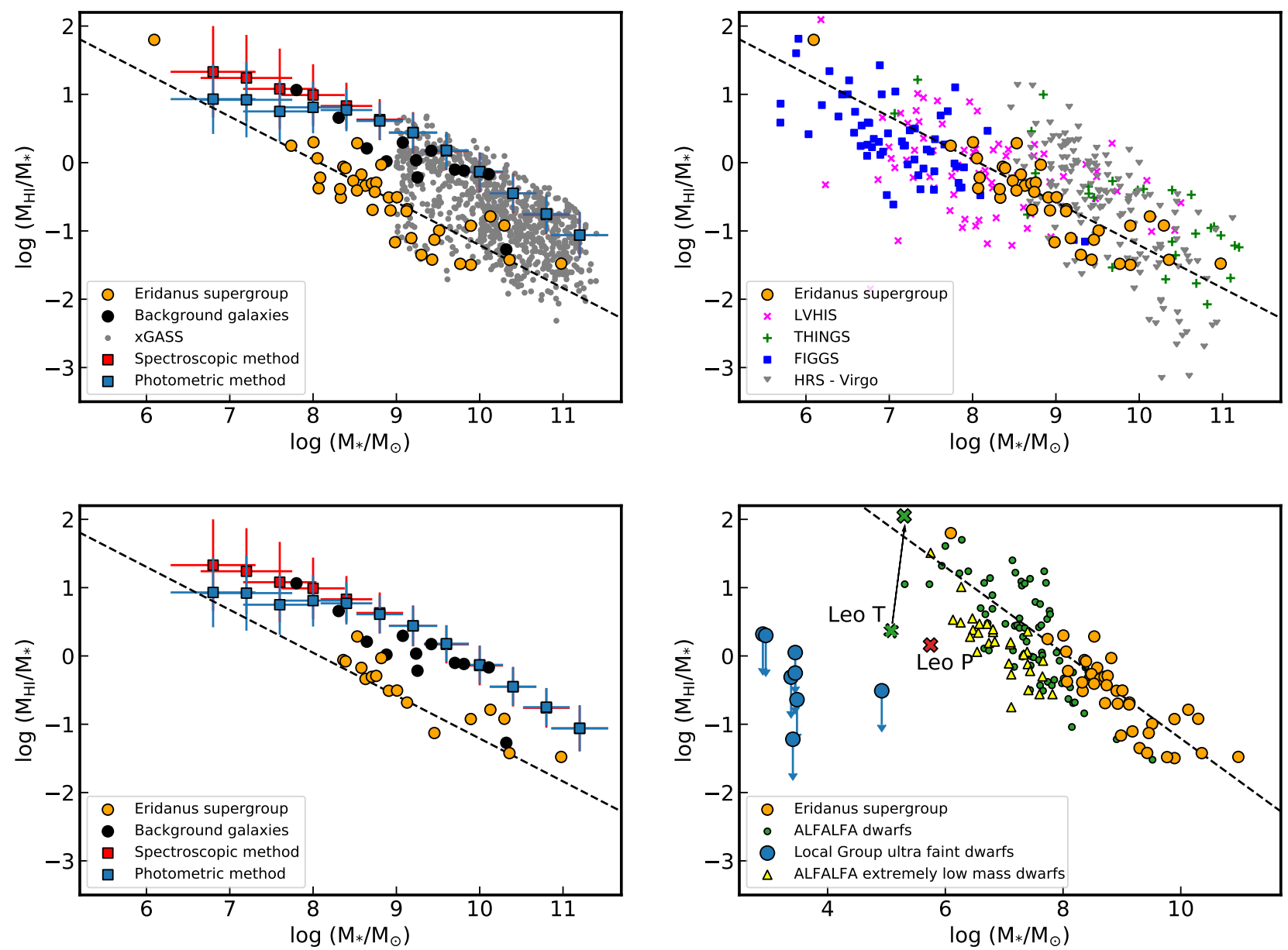

Figure 12. Atomic gas fraction scaling relation in logarithm scale $\left(\log \left(M_{\mathrm{HI}} / M_{*}\right)\right.$ versus $\left.\log M_{*}\right)$. Top left: Eridanus supergroup and background galaxies in this study are represented by orange and black dots, respectively. xGASS H I detected sample is in grey dots. The empirical relations derived from ALFALFA.40, SDSS spectroscopic (blue square) and photometric (red square) are overlaid on to the plot. Top right: LVHIS (magenta crosses), THINGS (green pluses), HRS - Virgo cluster members only (grey triangles) and FIGGS (blue squares) samples. The dashed line is used as a guide for the trend. Galaxies in the Eridanus supergroup (orange dots) are also overplotted and scaled to Chabrier IMF. Other studies used $H_{0}=70 \mathrm{~km} \mathrm{~s}^{-1} \mathrm{Mpc}^{-1}$, which the resulting $\mathrm{H}$ i and stellar masses are still within errors of our derived $M_{\mathrm{HI}} / M_{*}$ and $M_{*}$. Bottom left: Same as top left panel except with Eridanus supergroup galaxies that are detected at an assumed distance of $52 \mathrm{Mpc}$. Bottom right: Adding dwarf galaxies from ALFALFA.40 sub-sample (green dots), dwarf satellites within the Milky Way (blue circles), extremely low mass dwarfs from ALFALFA (yellow triangles), Leo T (green cross) and Leo P (red cross) for comparison. The shift of the Leo T data point is due to the use of a different $M_{\mathrm{HI}}$.

\section{ENVIRONMENT OF THE ERIDANUS SUPERGROUP}

The cosmic web is referred as a network of filaments with clustered galaxies and gases stretched across the Universe and separated by voids (Pomarède et al. 2017). It is seen in simulations and maps derived from galaxy redshift surveys. The so called the cosmic flow models are represented by the observed distribution of galaxies, peculiar velocities, and the underlying density field (see Tully et al. 2016; Pomarède et al. 2017 for detail on Cosmic V-web). In Figure 14, we show a 3-dimensional slice of the Universe centred at supergalactic position $\mathrm{SGX}=0$ and the environment that the Eridanus supergroup resides in. The model is computed using the overdensity field, $\Delta$, by the means of four iso-surface levels, namely $0.5,1.0,1.5$ and 2.0. We find that all 43 galaxies are located near the knot of Fornax. If we adopt a fixed $D_{\mathrm{L}}$ of $21 \mathrm{Mpc}$ for all galaxies, we also find that all of them fall in the same $\Delta=1.428$ grid. The eigenvalues $\lambda_{1}, \lambda_{2}$ and $\lambda_{3}$ of the shear tensor are $0.621,0.090$ and 0.030 , respectively. The positive eigenvalues suggest that they are residing in a knot cell of the V-web. However, the two lower eigenvalues $\left(\lambda_{2}, \lambda_{3}\right)$ indicate that some are edging toward the Sculptor Void. Overall, the model suggests that Eridanus supergroup is currently merging/falling onto the Fornax V-web knot. In future work, We will re-examine these findings with the derived individual distances from the Tully-Fisher relation paper.

\section{SUMMARY AND CONCLUSIONS}

We present the first WALLABY pre-pilot results from (almost) all the ASKAP antennas. These observations mimic the observation strategy for the WALLABY pilot survey. We assess the data quality by using a set of statistical metrics. We also perform source finding using sofIA and obtain a catalogue of $55 \mathrm{H}$ I sources within $\sim 37 \mathrm{MHz}$, including two H i clouds without stellar counterparts. In conjunction with deep optical images from the DR8 DESI Legacy Imaging Survey, we recover new gas-rich low surface brightness galaxies and dwarfs. 
Table 5. Star formation rates.

\begin{tabular}{|c|c|c|c|c|c|c|c|c|c|c|}
\hline ID & Designation & $\begin{array}{l}\mathrm{SFR}_{\mathrm{FUV}} \\
\left(\mathrm{M}_{\odot} \mathrm{yr}^{-1}\right) \\
(3)\end{array}$ & $\begin{array}{c}\sigma_{\mathrm{FUV}} \\
\left(\mathrm{M}_{\odot} \mathrm{yr}^{-1}\right) \\
(4)\end{array}$ & $\begin{array}{c}\mathrm{SFR}_{\mathrm{NUV}} \\
\left(\mathrm{M}_{\odot} \mathrm{yr}^{-1}\right) \\
(5)\end{array}$ & $\begin{array}{c}\sigma_{\mathrm{NUV}} \\
\left(\mathrm{M}_{\odot} \mathrm{yr}^{-1}\right) \\
(6)\end{array}$ & $\begin{array}{c}\mathrm{SFR}_{\mathrm{W} 4} \\
\left(\mathrm{M}_{\odot} \mathrm{yr}^{-1}\right) \\
(7)\end{array}$ & $\begin{array}{c}\sigma_{\mathrm{W} 4} \\
\left(\mathrm{M}_{\odot} \mathrm{yr}^{-1}\right) \\
(8)\end{array}$ & $\begin{array}{c}\mathrm{SFR}_{\text {total }} \\
\left(\mathrm{M}_{\odot} \mathrm{yr}^{-1}\right) \\
(9)\end{array}$ & $\begin{array}{c}\sigma_{\text {total }} \\
\left(\mathrm{M}_{\odot} \mathrm{yr}^{-1}\right) \\
(10)\end{array}$ & $\begin{array}{c}\log \mathrm{SFR} \\
\left(\mathrm{M}_{\odot} \mathrm{yr}^{-1}\right) \\
(11)\end{array}$ \\
\hline \multicolumn{11}{|c|}{ Eridanus Group } \\
\hline 1 & WALLABY J032831-222957 & 0.012 & 0.241 & 0.002 & 0.081 & $\ldots$ & $\ldots$ & 0.012 & 0.241 & -1.93 \\
\hline 2 & WALLABY J032900-220851 & $\ldots$ & $\ldots$ & $\ldots$ & $\ldots$ & 0.006 & 0.085 & $>0.006$ & 0.085 & $>-2.22$ \\
\hline 3 & WALLABY J032937-232103 & 0.015 & 0.101 & 0.004 & 0.075 & & & 0.015 & 0.101 & -1.82 \\
\hline 4 & WALLABY J032941-221642 & $\ldots$ & $\ldots$ & $\ldots$ & $\ldots$ & 0.050 & 0.070 & $>0.050$ & 0.070 & $>-1.30$ \\
\hline 5 & WALLABY J033019-210832 & $\ldots$ & $\ldots$ & $\ldots$ & $\ldots$ & 0.007 & 0.098 & $>0.007$ & 0.098 & $>-2.15$ \\
\hline 6 & WALLABY J033047-210333 & $\ldots$ & $\ldots$ & $\ldots$ & $\ldots$ & 0.046 & 0.070 & $>0.046$ & 0.070 & $>-1.34$ \\
\hline 7 & WALLABY J033228-232245 & 0.012 & 0.276 & 0.002 & 0.075 & $\ldots$ & $\ldots$ & 0.012 & 0.276 & -1.91 \\
\hline 8 & WALLABY J033257-210513 & $\ldots$ & $\ldots$ & $\ldots$ & $\ldots$ & 0.008 & 0.082 & $>0.008$ & 0.082 & $>-2.10$ \\
\hline 9 & WALLABY J033302-240756 & 0.034 & 0.090 & 0.006 & 0.067 & $\ldots$ & $\ldots$ & 0.034 & 0.090 & -1.47 \\
\hline 10 & WALLABY J033326-234246 & 0.057 & 0.077 & 0.014 & 0.067 & 0.127 & 0.069 & 0.184 & 0.017 & -0.74 \\
\hline 11 & WALLABY J033327-213352 & $\ldots$ & $\ldots$ & $\ldots$ & $\ldots$ & 0.270 & 0.069 & $>0.270$ & 0.069 & $>-0.57$ \\
\hline 12 & WALLABY J033341-212844 & $\ldots$ & $\ldots$ & $\ldots$ & $\ldots$ & 1.263 & 0.069 & $>1.263$ & 0.069 & $>0.101$ \\
\hline 13 & WALLABY J033408-232125 & $\ldots$ & $\ldots$ & 0.0002 & 0.4131 & $\ldots$ & $\ldots$ & 0.0002 & 0.4131 & -3.66 \\
\hline 14 & WALLABY J033501-245556 & 0.308 & 0.065 & 0.063 & 0.065 & 0.040 & 0.070 & 0.348 & 0.023 & -0.46 \\
\hline 15 & WALLABY J033527-211302 & $\ldots$ & $\ldots$ & $\ldots$ & $\ldots$ & 0.006 & 0.102 & $>0.006$ & 0.102 & $>-2.22$ \\
\hline 16 & WALLABY J033537-211742 & $\ldots$ & $\ldots$ & $\ldots$ & $\ldots$ & 0.004 & 0.093 & $>0.004$ & 0.093 & $>-2.40$ \\
\hline 17 & WALLABY J033617-253615 & 0.020 & 0.077 & 0.004 & 0.067 & 0.007 & 0.077 & 0.027 & 0.002 & -1.57 \\
\hline 18 & WALLABY J033653-245445 & 0.047 & 0.065 & 0.010 & 0.065 & 0.004 & 0.105 & 0.052 & 0.003 & -1.29 \\
\hline 19 & WALLABY J033723-235753* & $\cdots$ & $\ldots$ & $\ldots$ & $\ldots$ & $\ldots$ & $\ldots$ & $\ldots$ & $\ldots$ & $\ldots$ \\
\hline 20 & WALLABY J033728-243010 & 0.824 & 0.065 & 0.199 & 0.065 & 2.507 & 0.069 & 3.331 & 0.278 & 0.52 \\
\hline 21 & WALLABY J033854-262013 & 0.463 & 0.065 & 0.113 & 0.065 & $\ldots$ & $\ldots$ & 0.463 & 0.065 & -0.33 \\
\hline 22 & WALLABY J033911-222322* & $\ldots$ & $\ldots$ & $\ldots$ & $\ldots$ & $\ldots$ & $\ldots$ & $\ldots$ & $\ldots$ & $\ldots$ \\
\hline 23 & WALLABY J033921-212450 & $\ldots$ & $\ldots$ & 0.004 & 0.076 & 0.005 & 0.108 & 0.008 & 0.001 & -2.07 \\
\hline 24 & WALLABY J033941-235054 & 0.015 & 0.097 & 0.003 & 0.071 & $\ldots$ & $\ldots$ & 0.015 & 0.097 & -1.83 \\
\hline 25 & WALLABY J034036-213129 & $\ldots$ & $\ldots$ & 0.004 & 0.066 & $\ldots$ & $\ldots$ & 0.004 & 0.066 & -2.36 \\
\hline 26 & WALLABY J034040-221711 & 0.012 & 0.068 & 0.003 & 0.066 & $\ldots$ & $\ldots$ & 0.012 & 0.068 & -1.94 \\
\hline 27 & WALLABY J034056-223350 & 0.048 & 0.077 & 0.017 & 0.072 & 0.681 & 0.069 & 0.728 & 0.073 & -0.14 \\
\hline 28 & WALLABY J034057-214245 & $\ldots$ & $\ldots$ & 0.006 & 0.065 & $\ldots$ & $\ldots$ & 0.006 & 0.065 & -2.19 \\
\hline 29 & WALLABY J034114-235017 & 0.079 & 0.073 & 0.017 & 0.066 & 0.055 & 0.070 & 0.134 & 0.011 & -0.87 \\
\hline 30 & WALLABY J034131-214051 & 0.013 & 0.073 & 0.005 & 0.066 & 0.048 & 0.070 & 0.061 & 0.006 & -1.22 \\
\hline 31 & WALLABY J034219-224520 & 0.039 & 0.088 & 0.007 & 0.067 & $\ldots$ & $\ldots$ & 0.039 & 0.088 & -1.41 \\
\hline 32 & WALLABY J034337-211418 & 0.022 & 0.079 & 0.005 & 0.066 & $\ldots$ & $\ldots$ & 0.022 & 0.079 & -1.65 \\
\hline 33 & WALLABY J034434-211123 & 0.008 & 0.110 & 0.002 & 0.073 & $\ldots$ & $\ldots$ & 0.008 & 0.110 & -2.12 \\
\hline 34 & WALLABY J034456-234158 & $\ldots$ & $\ldots$ & 0.005 & 0.072 & 0.013 & 0.080 & 0.017 & 0.002 & -1.76 \\
\hline 35 & WALLABY J034517-230001 & $\ldots$ & $\ldots$ & $\ldots$ & $\ldots$ & 0.052 & 0.070 & $>0.052$ & 0.070 & $>-1.28$ \\
\hline 36 & WALLABY J034522-241208 & $\ldots$ & $\ldots$ & 0.001 & 1.138 & $\ldots$ & $\ldots$ & 0.001 & 1.138 & -3.04 \\
\hline 37 & WALLABY J034814-212824 & 0.058 & 0.069 & 0.013 & 0.066 & 0.060 & 0.070 & 0.119 & 0.009 & -0.93 \\
\hline
\end{tabular}

Note. This table is available in its entirety as Supporting Information with the electronic version of the paper. A portion is shown here for guidance regarding its form and content. *: H I clouds. Cols (1)-(2): Identification and designation. Cols (3)-(4): Star formation rate and its error in the GALEX FUV. Cols (5)-(6): Star formation rate and its error in the GALEX NUV. Cols (7)-(8): Star formation rate and its error in the neoWISE W4 band. Cols (9)-(10): Total star formation rate and its error. Col (11): Total star formation rate in the logarithmic scale.

We compare the integrated fluxes of WALLABY with the HIPASS and Parkes basketweave Eridanus survey. This reveals a 20\% deficit of integrated flux for the pre-pilot observations, which appears to arise from an accumulation of flux density scale errors, missing extended flux, low S/N ratio, and the use of inaccurate primary beam models for the edge beams. All of these are better addressed in the WALLABY pilot survey data. In the meantime, a uniform $20 \%$ correction is applied to the Eridanus fluxes for statistical purposes. Based on the galaxy group catalogues of G93, T15 and B06, we re-define membership of the Eridanus supergroup for our analysis. The mean recession velocity of the Eridanus group is $\sim 1500 \mathrm{~km} \mathrm{~s}^{-1}$.

We also perform $g$ and $r$-band photometry measurements using PROFOUND and derive the corresponding stellar mass based on the $M / L$-colour relation in Bell et al. (2003). By comparing with a more recently derived $M / L$-colour relation of Zibetti et al. (2009), we find that the stellar masses are generally consistent except for one low mass galaxy. We also calculate the $\mathrm{H}$ I masses and the $\mathrm{H}$ I deficiency parameter. The $\mathrm{H}_{\mathrm{I}}$ masses of Eridanus group and background galaxies range from $10^{7.5-9.8} \mathrm{M}_{\odot}$ and $10^{9-10.1} \mathrm{M}_{\odot}$, respectively. There are 20 galaxies in the Eridanus group considered to be $\mathrm{H}_{\mathrm{I}}$ deficient $\left(\mathrm{DEF}_{\mathrm{HI}}>0.3\right.$ dex $)$ and most of them are near other $\mathrm{H}_{\mathrm{I}}$ detected sources. All galaxies show signs of disturbance in their $\mathrm{H}$ I morphology. Both tidal interaction and ram-pressure stripping mechanisms contribute gas loss in the Eridanus group (Murugeshan et al. 2021).
There is no correlation between the $\mathrm{H}$ I deficiency parameter and the projected distance from the Eridanus group centre.

Two massive previously known $\mathrm{H}$ I clouds have been detected (see Wong et al. 2021). Smaller H I clouds are also detected as part of a single source detection. The most prominent tidal debris field is seen in NGC 1359 (an interacting pair) and NGC 1385. This debris has no optical counterpart. Some galaxies also show evidence of extra-planar gas extending out of the disk.

Comparing the gas fraction scaling relation with xGASS and the study of Maddox et al. (2015), we find that the gas fraction of background galaxies follows the general trend of decreasing $M_{\mathrm{HI}} / M_{*}$ with increasing $M_{*}$. However, the gas fraction of galaxies in the Eridanus supergroup is lower for a given $M_{*}$. To further investigate if the lower gas fraction trend is real in the Eridanus supergroup, we run a $\mathrm{H}_{\mathrm{I}}$ detection limit test. We find that a lower gas fraction trend remains for the galaxies in the Eridanus supergroup. We also compare the galaxies in the Eridanus supergroup with the gas fraction of dwarfs in the Local Group. The trend of $M_{\mathrm{HI}} / M_{*}$ versus $M_{*}$ among the Local Group dwarf population is not clear but an indication of various $\mathrm{H}$ I scaling relations are needed for different environment density.

To investigate if galaxies in our study are actively forming stars, we compare them with the star-forming main-sequence determined by S17, MG17 and MQ21. Overall, our galaxies in the Eridanus supergroup and background galaxies are actively forming stars. We rule 


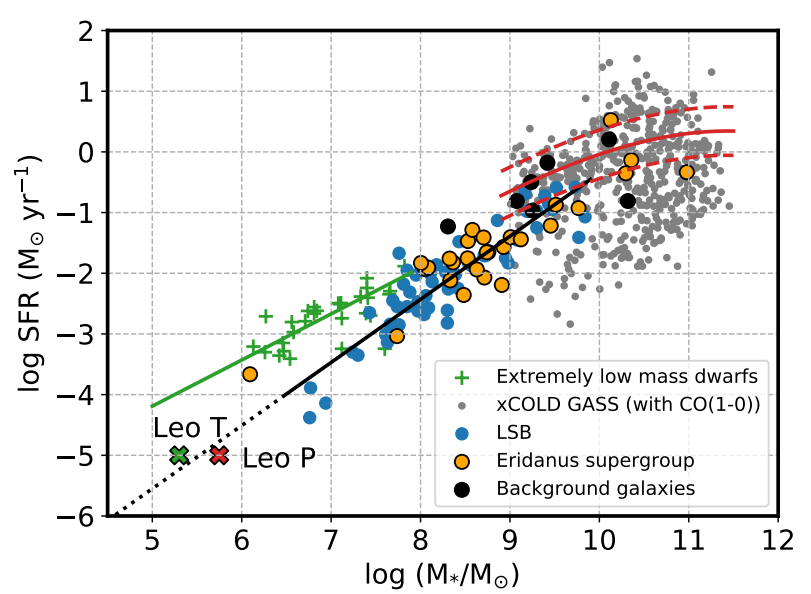

Figure 13. Star forming main sequence relation in logarithmic scale (log SFR vs $\log M_{*}\left(M_{*}\right)$. Eridanus supergroup and background galaxies in this study are represented by orange and black dots, respectively. xCOLD GASS (grey dots), LSB galaxies sample (blue dots), extremely low mass dwarfs (green pluses), Leo T (green cross) and Leo P (red cross) are also plotted for comparison. The solid black and dashed lines represent the fit and extended relation from MG17. The solid and dashed red lines represent the fit and \pm 0.4 dex scatter boundaries from S17. The green solid line represents the fit from MQ21.

out the possibility of a gas accreting event given that they generally follow the gas fraction scaling relation in the high mass regime.

\section{ACKNOWLEDGEMENTS}

This research was supported by the Australian Research Council Centre of Excellence for All Sky Astrophysics in 3 Dimensions (ASTRO 3D), through project number CE170100013. PK is partially supported by the BMBF project 05A17PC2 for D-MeerKAT. LVM acknowledges financial support from the grants AYA2015-65973C3-1-R and RTI2018-096228-B-C31 (MINECO/FEDER, UE), as well as from the State Agency for Research of the Spanish MCIU through the "Center of Excellence Severo Ochoa" award to the Instituto de Astrofisica de Andalucia (SEV-2017-0709). The Australian SKA Pathfinder is part of the Australia Telescope National Facility which is funded by the Australian Government with support from the National Collaborative Research Infrastructure Strategy and Industry Endowment Fund. ASKAP uses the resources of the Pawsey Supercomputing Centre with funding provided by the Australian Government under the National Computational Merit Allocation Scheme (project JA3) We acknowledge the Wajarri Yamatji as the traditional owners of the Murchison Radio Observatory (MRO) site and thank the operational staff onsite. This research has made use of images of the Legacy Surveys. The Legacy Surveys consist of three individual and complementary projects: the Dark Energy Camera Legacy Survey (DECaLS; Proposal ID \#2014B-0404; PIs: David Schlegel and Arjun Dey), the Beijing-Arizona Sky Survey (BASS; NOAO Prop. ID \#2015A-0801; PIs: Zhou Xu and Xiaohui Fan), and the Mayall z-band Legacy Survey (MzLS; Prop. ID \#2016A-0453; PI: Arjun Dey). DECaLS, BASS and MzLS together include data obtained, respectively, at the Blanco telescope, Cerro Tololo Inter-American Observatory, NSF's NOIRLab; the Bok telescope, Steward Observatory, University of Arizona; and the Mayall telescope, Kitt Peak National Observatory, NOIRLab. The Legacy Surveys project is hon- ored to be permitted to conduct astronomical research on Iolkam Du'ag (Kitt Peak), a mountain with particular significance to the Tohono O'odham Nation. BQF thanks A. Robotham and L. Davies for assisting the use ProFound and VISTAview cutout service.

\section{DATA AVAILABILITY}

The data underlying this article are available in the article and in its online supplementary material. The processed ASKAP data can be retrieved via CSIRO ASKAP Science Data Archive (CASDA) with a given scheduling block identification number. The DOI for the Eridanus data is https://dx.doi.org/10.25919/0yc5-f769. The data products from SOFIA is currently available within the WALLABY team and will be release to the public at a later date.

\section{REFERENCES}

Abramson A., Kenney J. D. P., Crowl H. H., Chung A., van Gorkom J. H., Vollmer B., Schiminovich D., 2011, AJ, 141, 164

Adams E. A. K., Oosterloo T. A., 2018, A\&A, 612, A26

Baker R. H., 1933, Annals of Harvard College Observatory, 88, 77

Barnes D. G., et al., 2001, MNRAS, 322, 486

Begum A., Chengalur J. N., Karachentsev I. D., Sharina M. E., Kaisin S. S., 2008, MNRAS, 386, 1667

Bekki K., Couch W. J., Ryan-Weber E. V., Webster R. L., 2004, in Duc P.-A., Braine J., Brinks E., eds, IAU Symposium Series Vol. 217, Recycling Intergalactic and Interstellar Matter. p. 418 (arXiv: astro-ph/0310358)

Bell E. F., McIntosh D. H., Katz N., Weinberg M. D., 2003, ApJS, 149, 289

Boselli A., Cortese L., Boquien M., Boissier S., Catinella B., Lagos C., Saintonge A., 2014, A\&A, 564, A66

Brough S., Forbes D. A., Kilborn V. A., Couch W., Colless M., 2006, MNRAS, 369,1351

Calzetti D., 2013, in Falcón-Barroso J., Knapen J. H., eds, Secular Evolution of Galaxies. p. 419

Cardelli J. A., Clayton G. C., Mathis J. S., 1989, ApJ, 345, 245

Catinella B., et al., 2018, MNRAS, 476, 875

Chabrier G., 2003, PASP, 115, 763

Chung A., van Gorkom J. H., Kenney J. D. P., Crowl H., Vollmer B., 2009, AJ, 138, 1741

Condon J. J., Cotton W. D., Greisen E. W., Yin Q. F., Perley R. A., Taylor G. B., Broderick J. J., 1998, AJ, 115, 1693

Cornwell T. J., 2008, IEEE Journal of Selected Topics in Signal Processing, 2, 793

Cortese L., et al., 2019, MNRAS, 485, 2656

Cortese L., Catinella B., Smith R., 2021, arXiv e-prints, p. arXiv:2104.02193

Dénes H., Kilborn V. A., Koribalski B. S., 2014, MNRAS, 444, 667

Dey A., et al., 2019, AJ, 157, 168

Dressler A., 1980, ApJ, 236, 351

Elagali A., et al., 2019, MNRAS, p. 1388

Escudero C. G., Faifer F. R., Smith Castelli A. V., Forte J. C., Sesto L. A., González N. M., Scalia M. C., 2018, MNRAS, 474, 4302

Fitzpatrick E. L., 1999, PASP, 111, 63

Fixsen D. J., Cheng E. S., Gales J. M., Mather J. C., Shafer R. A., Wright E. L., 1996, ApJ, 473, 576

For B. Q., Staveley-Smith L., Matthews D., McClure-Griffiths N. M., 2014, ApJ, 792, 43

For B. Q., et al., 2019, MNRAS, 489, 5723

Forbes D. A., Sánchez-Blázquez P., Phan A. T. T., Brodie J. P., Strader J., Spitler L., 2006, MNRAS, 366, 1230

Garcia A. M., 1993, A\&AS, 100, 47

Garcia A. M., Paturel G., Bottinelli L., Gouguenheim L., 1993, A\&AS, 98, 7

Giovanelli R., et al., 2005, AJ, 130, 2598

Gómez P. L., et al., 2003, ApJ, 584, 210

Graziani R., Courtois H. M., Lavaux G., Hoffman Y., Tully R. B., Copin Y., Pomarède D., 2019, MNRAS, 488, 5438 


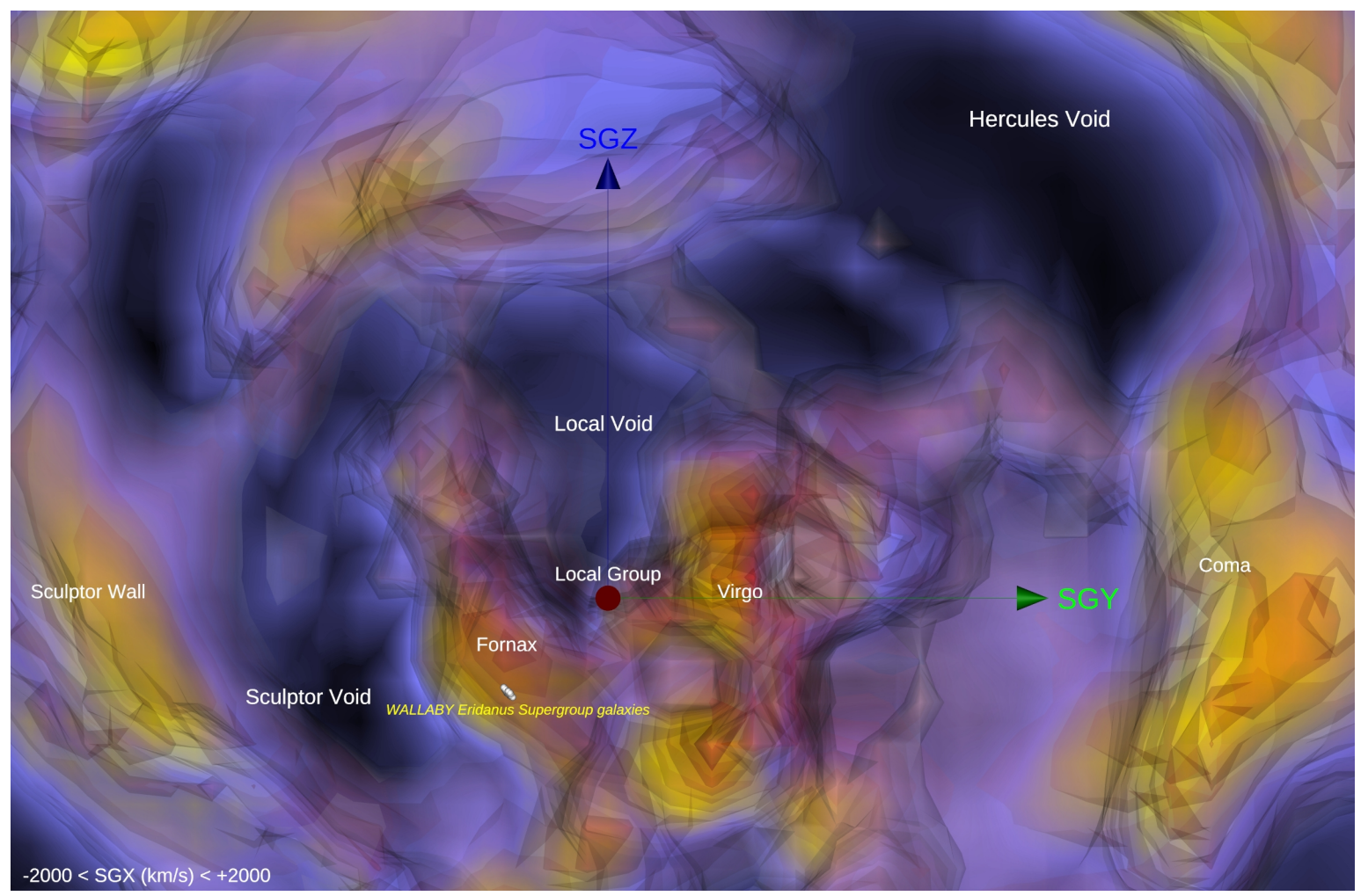

Figure 14. A slice of 3-dimensional model contains planes within the $\mathrm{SGX}=-2000 \mathrm{~km} \mathrm{~s}^{-1}$ and SGX=+2000 km s${ }^{-1}$ is shown face-on from the positive SGX direction. The positions of the Eridanus supergroup galaxies are plotted against a map of the density contrast $\delta$ reconstructed from the Cosmicflows-3 Catalog of peculiar velocities (Graziani et al. 2019). This map is obtained with the ray-casting volume rendering technique, resulting in a smooth representation of the $\delta$ field ranging from most underdense (deep blue color) to most overdense (yellow), combined with a series of semi-transparent iso-surface polygons resulting in a sharp materialization of the surfaces ranging from $\delta=0$ (grey surface) to highly overdense ( $\delta=2.8$ in red). The grey surface delineates the frontier between underdense and overdense patches of the local Universe. Scale and orientation are provided by the $5000 \mathrm{~km} \mathrm{~s}^{-1}$ long arrows emanating from our position and associated with the cardinal axes of the supergalactic coordinate system. An interactive 3-dimensional model is available at https: //sketchfab.com/3d-models/wallaby-eridanus-supergroup-vs-cf3-density-2d92e8a9f4b74f4293d9fabb9a6e73b3.

Grcevich J., Putman M. E., 2009, ApJ, 696, 385

Haynes M. P., Giovanelli R., 1984, AJ, 89, 758

Hibbard J. E., van Gorkom J. H., 1996, AJ, 111, 655

Hickson P., 1997, ARA\&A, 35, 357

Hotan A. W., et al., 2021, Publ. Astron. Soc. Australia, 38, e009

Hoyle B., Masters K. L., Nichol R. C., Jimenez R., Bamford S. P., 2012, MNRAS, 423, 3478

Huang S., Haynes M. P., Giovanelli R., Brinchmann J., Stierwalt S., Neff S. G., 2012, AJ, 143, 133

Johnston S., et al., 2007, Publ. Astron. Soc. Australia, 24, 174

Jones D. H., et al., 2004, MNRAS, 355, 747

Jones M. G., et al., 2018, A\&A, 609, A17

Katz N., Keres D., Dave R., Weinberg D. H., 2003, in Rosenberg J. L., Putman M. E., eds, Astrophysics and Space Science Library Vol. 281, The IGM/Galaxy Connection. The Distribution of Baryons at $\mathrm{z}=0$. p. 185 (arXiv : astro-ph/0209279), doi:10.1007/978-94-010-0115-1_34

Kenney J. D. P., van Gorkom J. H., Vollmer B., 2004, AJ, 127, 3361

Kereš D., Katz N., Weinberg D. H., Davé R., 2005, MNRAS, 363, 2

Kilborn V. A., Forbes D. A., Koribalski B. S., Brough S., Kern K., 2006, MNRAS, 371, 739

Kilborn V. A., Forbes D. A., Barnes D. G., Koribalski B. S., Brough S., Kern K., 2009, MNRAS, 400, 1962

Kleiner D., et al., 2019, MNRAS, p. 2001

Koribalski B. S., et al., 2018, MNRAS, 478, 1611

Koribalski B. S., et al., 2020, Ap\&SS, 365, 118

Kundu A., Whitmore B. C., 2001, AJ, 122, 1251

Lee-Waddell K., et al., 2019, MNRAS, 487, 5248

Li J., Obreschkow D., Lagos C., Cortese L., Welker C., Džudžar R., 2020,
MNRAS, 493, 5024

Madau P., Dickinson M., 2014, ARA\&A, 52, 415

Maddox N., Hess K. M., Obreschkow D., Jarvis M. J., Blyth S. L., 2015, MNRAS, 447, 1610

Mahajan S., 2013, MNRAS, 431, L117

Marinoni C., Monaco P., Giuricin G., Costantini B., 1998, ApJ, 505, 484

Martin D. C., et al., 2005, ApJ, 619, L1

Masci F. J., Fowler J. W., 2009, in Bohlender D. A., Durand D., Dowler P., eds, Astronomical Society of the Pacific Conference Series Vol. 411, Astronomical Data Analysis Software and Systems XVIII. p. 67 (arXiv:0812.4310)

Materne J., 1978, A\&A, 63, 401

McConnell D., et al., 2016, Publ. Astron. Soc. Australia, 33, e042

McConnell D., et al., 2020, Publ. Astron. Soc. Australia, 37, e048

McGaugh S. S., Schombert J. M., Lelli F., 2017, ApJ, 851, 22

McQuinn K. B. W., et al., 2015, ApJ, 812, 158

McQuinn K. B. W., et al., 2021, arXiv e-prints, p. arXiv:2105.05100

Meyer M. J., et al., 2004, MNRAS, 350, 1195

Meyer M., Robotham A., Obreschkow D., Westmeier T., Duffy A. R., Staveley-Smith L., 2017, Publ. Astron. Soc. Australia, 34

Morshidi-Esslinger Z., Davies J. I., Smith R. M., 1999, MNRAS, 304, 311

Murugeshan C., et al., 2021, MNRAS

Obreschkow D., Glazebrook K., Kilborn V., Lutz K., 2016, ApJ, 824, L26

Omar A., Dwarakanath K. S., 2005a, Journal of Astrophysics and Astronomy, 26,1

Omar A., Dwarakanath K. S., 2005b, Journal of Astrophysics and Astronomy, 26,71

Paturel G., Bottinelli L., Fouque P., Gouguenheim L., 1988, in European 
Southern Observatory Conference and Workshop Proceedings. pp 435440

Pisano D. J., 2014, AJ, 147, 48

Pomarède D., Hoffman Y., Courtois H. M., Tully R. B., 2017, ApJ, 845, 55 Postman M., et al., 2005, ApJ, 623, 721

Putman M. E., Zheng Y., Price-Whelan A. M., Grcevich J., Johnson A. C., Tollerud E., Peek J. E. G., 2021, arXiv e-prints, p. arXiv:2101.07809

Reynolds T. N., et al., 2019, MNRAS, 482, 3591

Richtler T., 2003, The Globular Cluster Luminosity Function: New Progress in Understanding an Old Distance Indicator. Lecture Notes in Physics, Springer, pp 281-305, doi:10.1007/978-3-540-39882-0_15

Robotham A. S. G., Davies L. J. M., Driver S. P., Koushan S., Taranu D. S., Casura S., Liske J., 2018, MNRAS, 476, 3137

Ryan-Weber E., Webster R., Bekki K., 2003, Galactic Recycling: The HI Ring Around NGC 1533. Kluwer, p. 223, doi:10.1007/978-94-010-0115-1_40

Ryan-Weber E. V., Begum A., Oosterloo T., Pal S., Irwin M. J., Belokurov V., Evans N. W., Zucker D. B., 2008, MNRAS, 384, 535

Saintonge A., et al., 2017, ApJS, 233, 22

Salpeter E. E., 1955, ApJ, 121, 161

Schlafly E. F., Finkbeiner D. P., 2011, ApJ, 737, 103

Schlegel D. J., Finkbeiner D. P., Davis M., 1998, ApJ, 500, 525

Serra P., Jurek R., Flöer L., 2012, Publ. Astron. Soc. Australia, 29, 296

Serra P., et al., 2015, MNRAS, 448, 1922

Simard L., et al., 2009, A\&A, 508, 1141

Solanes J. M., Giovanelli R., Haynes M. P., 1996, ApJ, 461, 609

Sorgho A., et al., 2019, MNRAS, 482, 1248

Spergel D. N., et al., 2007, ApJS, 170, 377

Springob C. M., Haynes M. P., Giovanelli R., Kent B. R., 2005, ApJS, 160, 149

Tully R. B., 1980, ApJ, 237, 390

Tully R. B., 2015, AJ, 149, 171

Tully R. B., Courtois H. M., Sorce J. G., 2016, AJ, 152, 50

Verdes-Montenegro L., Yun M. S., Williams B. A., Huchtmeier W. K., Del Olmo A., Perea J., 2001, A\&A, 377, 812

Walter F., Brinks E., de Blok W. J. G., Bigiel F., Kennicutt Robert C. J., Thornley M. D., Leroy A., 2008, AJ, 136, 2563

Wang J., et al., 2017, MNRAS, 472, 3029

Waugh M., 2005, PhD thesis, University of Melbourne

Westmeier T., Staveley-Smith L., Calabretta M., Jurek R., Koribalski B. S., Meyer M., Popping A., Wong O. I., 2015, MNRAS, 453, 338

Westmeier T., et al., 2017, MNRAS, 472, 4832

Westmeier T., et al., 2021, MNRAS,

Whiting M. T., 2020, in Ballester P., Ibsen J., Solar M., Shortridge K., eds, Astronomical Society of the Pacific Conference Series Vol. 522, Astronomical Data Analysis Software and Systems XXVII. p. 469

Wieringa M., Raja W., Ord S., 2020, in Pizzo R., Deul E. R., Mol J. D., de Plaa J., Verkouter H., eds, Astronomical Society of the Pacific Conference Series Vol. 527, Astronomical Society of the Pacific Conference Series. p. 591

Willmer C. N. A., 2018, ApJS, 236, 47

Willmer C. N. A., Focardi P., da Costa L. N., Pellegrini P. S., 1989, AJ, 98, 1531

Wolfinger K., Kilborn V. A., Ryan-Weber E. V., Koribalski B. S., 2016, Publ. Astron. Soc. Australia, 33, e038

Wong O. I., et al., 2021, MNRAS

Zabludoff A. I., Mulchaey J. S., 1998, ApJ, 496, 39

Zibetti S., Charlot S., Rix H.-W., 2009, MNRAS, 400, 1181

da Costa L. N., et al., 1988, ApJ, 327, 544

de Vaucouleurs G., 1975, Nearby Groups of Galaxies. University of Chicago Press, p. 557

\section{SUPPORTING INFORMATION}

Additional Supporting Information can be found in the online version of this article.

Figure 5: Integrated $\mathrm{H}$ I column density maps of individual sources overlaid onto the DR8 DESI Legacy Imaging Survey $g$-band stacked images. Velocity field maps and spectrum of individual sources.

Table 3: Source catalogue and derived parameters.

Table 4: Photometry, morphology and derived parameters.

Table 5: Star formation rates.

\section{APPENDIX A: H I AND OPTICAL MORPHOLOGIES}

In this section, we examine the $\mathrm{H}_{\mathrm{I}}$ and optical morphologies of galaxies. We refer the reader to Figures 4 and 5 for $\mathrm{H}_{\mathrm{I}}$ optical morphologies and ID number of galaxies. All of the galaxies in the Eridanus supergroup show signs of disturbance in H I morphology. The $\mathrm{H}$ I detection of NGC 1347 (\#4) includes its interacting galaxy, PGC 816443. An H I extension is visible at $N_{\mathrm{HI}} \sim 5 \times 10^{20} \mathrm{~cm}^{-2}$. LEDA 832131 (\#5) does not have a morphological classification in NED. We identify it as an irregular dwarf with multiple starforming regions visible in the optical image. It is located to the south-west of both ESO 548-G029 (\#6) and ESO 548-G034 (\#8), which are also distorted in $\mathrm{H}_{\mathrm{I}}$ morphology. This indicates that the three are likely interacting with each other. ESO 548-G036 (\#11) is located south-west of IC 1953 (\#12). There is strong evidence that these two galaxies are interacting with an $\mathrm{HI}$ extension seen in IC 1953 towards ESO 548-G036. An H I cloud is also detected north of ESO 548-G036. NGC 1385 (\#20) is a face-on spiral galaxy that is located $0.6^{\circ}$ south of WALLABY J033723-235753. Its H I morphology is very distorted and there is a southern tidal debris field. There is no optical counterpart for the H I debris. ESO 482-G035 (\#29) is a face-on SBab galaxy with a distorted and elongated $\mathrm{H}_{\mathrm{I}}$ morphology, which is in contrast to the non-fully developed H I disk as shown in Omar \& Dwarakanath (2005b). There is also a known LSB dwarf, F482-018 (Morshidi-Esslinger et al. 1999), south of ESO 482-G035, which does not have an H I detection. Given the elongation of the $\mathrm{H}_{\mathrm{I}}$, this dwarf could potentially be a satellite of ESO 482-G035. GALEXASC J033408.06-232130.1 (\#13; DEF ${ }_{\mathrm{HI}}$ $=-0.83)$ is a LSB dwarf and we find a H I cloud is seen on the eastern side of this galaxy. ESO 482-G005 (\#9; $\left.\mathrm{DEF}_{\mathrm{HI}}=-0.29\right)$ is an edge-on galaxy. Both galaxies show signatures of disturbance.

NGC 1422 (\#30) shows a truncated H I disk that is smaller than the stellar disk. LEDA 135119 (\#33) is classified as a spiral galaxy in NED but no distinctive spiral arms are seen in our optical image. Its $\mathrm{H}_{\text {I }}$ morphology is asymmetric and an $\mathrm{H}$ i cloud north of the galaxy is detected. It is located at the edge of the observed field, and hence, we cannot rule out the possibility that the galaxy is interacting with another nearby galaxy. Both NGC 1398 (\#21) and NGC 1415 (\#27) are large bright galaxies with $B$-band optical isophotal diameter measured at $25 \mathrm{mag} \operatorname{arcsec}^{-2}$ of $425^{\prime \prime}$ and $208^{\prime \prime}$, respectively. The H I traces various star-forming sites and is extended beyond the optical disk. NGC 1398 (SBab galaxy) also has a ring like $\mathrm{H}$ i that resembles a similar feature as seen in NGC 1533 (Ryan-Weber et al. 2003). Modeling of NGC 1533 suggests that the ring like $\mathrm{H}_{\mathrm{I}}$ is formed as a result of an unequal-mass merging event between gas-rich LSB galaxies and host SB galaxy's disk (Bekki et al. 2004). NGC 1398 is located at the edge of the observed field and furthest from the Eridanus group centre. NGC 1438 (\#35) is a SB0/a galaxy that has a peculiar $\mathrm{H}$ I morphology. There are two tidal tails south of the galaxy and no optical counterpart has been identified along the tails.

Other interesting $\mathrm{H}$ I morphologies within the Eridanus group include ESO 482-G013 (\#18) and ESO 482-G027 (\#24). There are $\mathrm{H}_{\mathrm{I}}$ extensions seen above and below the $\mathrm{H}_{\mathrm{I}}$ disk of ESO 482-G013 (\#18). Some edge-on galaxies also show evidence of extraplanar gas with plumes of gas extended out of the disk (e.g. IC 1952, 
ESO 482-G013 and ESO 482-G011). The latter is an irregular galaxy and its $\mathrm{H}_{\mathrm{I}}$ morphology resembles a head-tail structure with the tail pointing away from the Eridanus group centre. It is also located $0.53^{\circ}$ and $1.46^{\circ}$ angular distance from the two massive Hi clouds, WALLABY J033723-235753 (\#19) and WALLABY J033911-222322 (\#22). We refer the reader to Wong et al. (2021) for the discussion of the origin of these two H I clouds.

Among the three detections in the NGC 1407 group, a large and spectacular Hi tidal debris field is seen around the NGC 1359, an interacting galaxy pair (\#41). Both ESO 548-G065 (\#43) and NGC 1390 (\#42) show disruption of the H I outer disk. Within the NGC 1332 group, NGC 1325 (\#38) is a SAbc galaxy that forms a pair along with NGC 1325A. There is no H I detection of NGC 1325A in our study but there is in W15. Evidence of interaction is seen with gas extending from the north corner of LSB galaxy, ESO 548-G011 (\#39), towards NGC 1325.

The $\mathrm{H}_{\mathrm{I}}$ morphology of all background galaxies also shows signs of disturbance. ESO 549-G023 (\#50) is a SBa galaxy and is also classified as an emission line galaxy. It has an extended $\mathrm{H}_{\mathrm{I}}$ "tail" toward the south-east and some small $\mathrm{H}$ I debris to the north-east of the galaxy. LEDA 798516 (\#48) is a LSB galaxy and is possibly interacting with a few galaxies south of it, including LEDA 798377 (unresolved). Another $\mathrm{H}_{\mathrm{I}}$ detection is also seen at the north-east side of LEDA 798516. The location coincides with an edge-on galaxy (GALEXASC J033838.83-233810.9), which is not identified as a separate source by soFIA.

This paper has been typeset from a $\mathrm{T}_{\mathrm{E}} \mathrm{X} / \mathrm{LAT} \mathrm{E} \mathrm{X}$ file prepared by the author. 